\title{
Rotation- and temperature-dependence of stellar latitudinal differential rotation $\star, \star \star$
}

\author{
A. Reiners $s^{1,2, \star \star \star}$ \\ 1 Astronomy Department, 521 Campbell Hall, University of California, Berkeley, CA 94720, USA \\ e-mail: areiners@astron.berkeley.edu \\ 2 Hamburger Sternwarte, Universität Hamburg, Gojenbergsweg 112, 21029 Hamburg, Germany
}

Received 25 July 2005 / Accepted 12 September 2005

\begin{abstract}
More than 600 high resolution spectra of stars with spectral type $\mathrm{F}$ and later were obtained in order to search for signatures of differential rotation in line profiles. In 147 stars the rotation law could be measured, with 28 of them found to be differentially rotating. Comparison to rotation laws in stars of spectral type A reveals that differential rotation sets in at the convection boundary in the HR-diagram; no star that is significantly hotter than the convection boundary exhibits the signatures of differential rotation. Four late A-/early F-type stars close to the convection boundary and at $v \sin i \approx 100 \mathrm{~km} \mathrm{~s}^{-1}$ show extraordinarily strong absolute shear at short rotation periods around one day. It is suggested that this is due to their small convection zone depth and that it is connected to a narrow range in surface velocity; the four stars are very similar in $T_{\text {eff }}$ and $v \sin i$. Detection frequencies of differential rotation $\alpha=\Delta \Omega / \Omega>0$ were analyzed in stars with varying temperature and rotation velocity. Measurable differential rotation is more frequent in late-type stars and slow rotators. The strength of absolute shear, $\Delta \Omega$, and differential rotation $\alpha$ are examined as functions of the stellar effective temperature and rotation period. The highest values of $\Delta \Omega$ are found at rotation periods between two and three days. In slower rotators, the strongest absolute shear at a given rotation rate $\Delta \Omega_{\max }$ is given approximately by $\Delta \Omega_{\max } \propto P^{-1}$, i.e., $\alpha_{\max } \approx$ const. In faster rotators, both $\alpha_{\max }$ and $\Delta \Omega_{\max }$ diminish less rapidly. A comparison with differential rotation measurements in stars of later spectral type shows that F-stars exhibit stronger shear than cooler stars do and the upper boundary in absolute shear $\Delta \Omega$ with temperature is consistent with the temperature-scaling law found in Doppler Imaging measurements.
\end{abstract}

Key words. stars: activity - stars: late-type - stars: rotation - stars: general

\section{Introduction}

Stellar rotation rates range from those too slow to be detected by Doppler broadening up to rates at which centrifugal forces become comparable to surface gravity. Surface magnetic fields, either fossil or generated by some type of magnetic dynamo, can couple to ionized plasma and brake a star's rotation via a magneto-thermal wind. Magnetic braking is observed in stars with deep convective envelopes where magnetic dynamo processes can efficiently maintain strong magnetic fields. As a consequence, field stars of a spectral type later than $\mathrm{F}$ are generally slow rotators with surface velocities below $10 \mathrm{~km} \mathrm{~s}^{-1}$.

Magnetic braking requires the existence of a magnetic field, which also causes the plethora of all the effects found in stellar

* Based on observations carried out at the European Southern Observatory, Paranal and La Silla, PIDs 68.D-0181, 69.D-0015, 71.D-0127, 72.D-0159, 73.D-0139, and on observations collected at the Centro Astronómico Hispano Alemán (CAHA) at Calar Alto, operated jointly by the Max-Planck Institut für Astronomie and the Instituto de Astrofísica de Andalucía (CSIC).

$\star \star$ Appendices A and B are only available in electronic form at http://www. edpsciences.org

$\star \star \star$ Marie Curie Outgoing International Fellow. magnetic activity. While early magnetic braking may be due to fossil fields amplified during contraction of the protostellar cloud, magnetic activity at later phases requires a mechanism that maintains magnetic fields on longer timescales. In the Sun, a magnetic dynamo located at the interface between convective envelope and radiative core is driven by radial differential rotation. This dynamo has been identified as the main source of magnetic fields, although there is growing evidence that it is not the only source of magnetic field generation (e.g., Schrijver \& Zwaan 2000) and that magnetic fields also exist in fully convective stars (Johns-Krull \& Valenti 1996). The solar-type dynamo, however, has the potential to generate magnetic fields in all non-fully convective stars, as long as they show a convective envelope, and magnetic fields have been observed in a variety of slowly rotating stars (e.g., Marcy 1984; Gray 1984; Solanki 1991).

Calculations of stellar rotation laws, which describe angular velocity as a function of radius and latitude, have been carried out by Kitchatinov \& Rüdiger (1999) and Küker \& Rüdiger (2005) for different equatorial angular velocities. Kitchatinov \& Rüdiger (1999) investigated rotation laws in a G2- and a K5-dwarf. They expect stronger latitudinal 
differential rotation in slower rotators, and their G2-dwarf model exhibits stronger differential rotation than the K5-dwarf does. Küker \& Rüdiger (2005) calculate a solar-like model, as well as a model of an F8 main sequence star. They also come to the conclusion that differential rotation is stronger in the hotter model; the maximum differential rotation in the F8 star is roughly twice as strong as in the G2 star for the same viscosity parameter. The calculated dependence of horizontal shear on rotation rate, however, does not show a monotonic slope but has a maximum that occurs near $10 \mathrm{~d}$ in the F8 type star and around $25 \mathrm{~d}$ in the solar-type star. The strength of differential rotation depends on the choice of the viscosity parameter, which is not well constrained, but the trends in temperature and rotation are unaffected by that choice.

Observational confirmation of solar radial and latitudinal differential rotation comes from helioseismological studies that provide a detailed picture of the differentially rotating outer convection zone (e.g., Schou 1998). Such seismological studies are not yet available for any other star. Asteroseismological missions, like COROT and Kepler, may open a new window on stellar differential rotation, but its data quality may provide only a very limited picture in the near future (Gizon \& Solanki 2004). In the case of the Sun, radial differential rotation manifests itself in latitudinal differential rotation that can be observed at the stellar surface, but all stars except the Sun are at distances where their surfaces cannot be adequately resolved. With the advent of large optical interferometers, more may be learned from observations of spatially resolved stellar surfaces (Domiciano de Souza et al. 2004). For now, we have to rely on indirect methods to measure the stellar rotation law.

Photometric programs that search for stellar differential rotation assume that starspots emerge at various latitudes with different rotation rates, as observed on the Sun. Hall (1991) and Donahue et al. (1996) measured photometric rotation periods and interpreted seasonal variations as the effect of differential rotation on migrating spots. Although these techniques are comparable to the successful measurements of solar differential rotation through sunspots (Balthasar et al. 1986), they still rely on a number of assumptions, e.g., the spot lifetime being longer than the observational sequence, an assumption difficult to test in stars other than the Sun (cf. Wolter et al. 2005). Photometric measurements report lower limits for differential rotation on the order of $10 \%$ of the rotation velocity (i.e. the equator rotating $10 \%$ faster than the polar regions), and differential rotation is reported to be stronger in slower rotators.

Doppler Imaging (DI) has been extensively used to determine latitudinal differential rotation where the derived maps are constructed from time-series of high-resolution spectra. Differential rotation can then be extracted from comparing two surface maps taken with time separation of a few rotation periods (e.g., Donati \& Collier Cameron 1997; Wolter et al. 2005). Doppler maps can also be constructed that incorporate differential rotation during the inversion algorithm (Petit et al. 2002). Detections of differential rotation through DI have recently been compiled by Barnes et al. (2005), who also analyze dependence on stellar rotation and temperature, and then compare them to results obtained from other techniques. Their results will be discussed in Sect. 6 .
The technique employed in this paper is to search for latitudinal differential rotation in the shape of stellar absorption line profiles. This method is applicable only to stars not dominated by spots. From a single exposure, latitudinal solar-like differential rotation - i.e. the equator rotating faster than polar regions - can be derived by measuring its unambiguous fingerprints in the Fourier domain. The foundations of the Fourier transform method (FTM) were laid by Gray $(1977,1998)$, and first models were done by Bruning (1981). A detailed description of the fingerprints of solar-like differential rotation and the first successful detections are given in Reiners \& Schmitt (2002a, 2003a). The FTM is limited to moderately rapid rotators (see Sect. 2), but the big advantage of this method is that latitudinal differential rotation can be measured from a single exposure. This allows the analysis of a large sample of stars with a comparably small amount of telescope time. Reiners \& Schmitt (2003a) report on differential rotation in ten out of a sample of 32 stars of spectral types F0-G0 with projected rotation velocities $12 \mathrm{~km} \mathrm{~s}^{-1}<v \sin i<50 \mathrm{~km} \mathrm{~s}^{-1}$. Reiners \& Schmitt (2003b) investigated a sample of 70 rapid rotators with $v \sin i>45 \mathrm{~km} \mathrm{~s}^{-1}$ and found a much lower fraction of differential rotators. Differential rotation has also been sought in A-stars that have no deep convective envelopes. Reiners \& Royer (2004) report on three objects out of 76 in the range $\mathrm{A} 0-\mathrm{F} 1,60 \mathrm{~km} \mathrm{~s}^{-1}<v \sin i<150 \mathrm{~km} \mathrm{~s}^{-1}$, which show signatures of differential rotation.

In the cited works, differential rotation is investigated in stars of limited spectral types and rotation velocities. In this paper, I aim to investigate all measurements of differential rotation from FTM, add new observations, and finally compare them to results from DI and theoretical predictions. Currently, more than 600 stars were observed during the course of this project, and in 147 of them the rotation law could be measured successfully.

\section{Differential rotation in line profiles}

Latitudinal differential rotation has a characteristic fingerprint in the shape of the rotational broadening that appears in each spectral line. Since all other line broadening mechanisms, like turbulence, thermal, and pressure broadening, etc., also affect the shape of spectral lines, the effects of differential rotation are very subtle. Signatures of differential rotation and a recipe for measuring them are presented in Reiners \& Schmitt (2002a). Since the signatures are so small, and spectral line blending is a serious issue even in stars of moderate rotation rates at $v \sin i \approx$ $20 \mathrm{~km} \mathrm{~s}^{-1}$, single lines are not measured, but instead a total broadening function is constructed from many lines of similar intrinsic shape. This process typically involves 15 lines in slow rotators observed at very high resolution (Sect. 3), and 300 lines in rapid rotators observed at lower resolution. In order to derive a unique broadening function at the required precision, high data quality is required. Detailed information about demands on data quality can be found in Reiners \& Schmitt (2003a,b).

Interpretation of the profile's shape with rotational broadening requires that the line profiles are not affected by starspots, stellar winds, spectroscopic multiplicity, etc. The Fourier transform method is therefore limited to unspotted single stars with 
projected rotation velocities $v \sin i \gtrsim 12 \mathrm{~km} \mathrm{~s}^{-1}$, and an upper limit at $v_{\mathrm{eq}} \approx 200 \mathrm{~km} \mathrm{~s}^{-1}$ is set by gravitational darkening. More specific information on the influence of starspots, of very rapid rotation, and some examples of detected signatures of differential rotation in line profiles can be found in Reiners (2003), and Reiners \& Schmitt (2002a, 2003a,b).

From the derived broadening profile, the rotation law is determined by measuring the first two zeros of the profile's Fourier transform, $q_{1}$ and $q_{2}$. In sufficiently rapid rotators, those are direct indicators of rotational broadening since other broadening mechanisms (for example turbulence or instrumental broadening) do not show zeros at such low frequencies. This is also the reason only stars with $v \sin i \gtrsim 12 \mathrm{~km} \mathrm{~s}^{-1}$ can be studied; in slower rotators, line broadening is dominated by turbulence and the zeros due to rotation cannot be measured. In stars spinning fast enough to be analyzed with FTM, approximation of net broadening by convolutions is also justified. The important point in choosing the Fourier domain for profile analysis is that convolutions become multiplications in Fourier space. Thus, the fingerprints of rotational broadening are directly visible in the observed broadening profile's Fourier transform, and the spectra do not have to be corrected for instrumental or for any other line broadening, as long as the targets' rotation dominates the important frequency range.

In the following, the stellar rotation law will be approximated in analogy to the solar case. Differential rotation is expressed in terms of the variable $\alpha$, with $\Omega$ the angular velocity and $l$ the latitude. The rotation law is approximated by

$$
\begin{aligned}
\Omega(l) & =\Omega_{\text {Equator }} \cdot \alpha \sin ^{2}(l), \\
\alpha & =\frac{\Omega_{\text {Equator }}-\Omega_{\text {Pole }}}{\Omega_{\text {Equator }}}=\frac{\Delta \Omega}{\Omega} .
\end{aligned}
$$

Solar-like differential rotation is characterized by $\alpha>0\left(\alpha_{\odot} \approx\right.$ $0.2)$. Sometimes $\Delta \Omega$ is called absolute differential rotation. To avoid confusion with relative differential rotation $\alpha=\Delta \Omega / \Omega$, I will refer to $\Delta \Omega$ as absolute shear and to $\alpha$ as differential rotation. $\Delta \Omega$ is given in units of $\operatorname{rad~d}^{-1}$. As shown in Reiners \& Schmitt (2003a), the parameter $\alpha / \sqrt{\sin i}$, with $i$ the inclination of the stellar rotation axis, can be directly obtained from the ratio of the Fourier transform's first two zeros, $q_{2} / q_{1}$. Thus, determination of the stellar rotation law in Eq. (1) from a single stellar spectrum is a straightforward exercise.

\subsection{Variables to describe differential rotation}

The role of differential rotation especially for magnetic field generation in rapid rotators is not understood well. In the case of the Sun, we know that its magnetic cycle is driven, at least in part, by radial differential rotation, which itself is reflected in latitudinal differential rotation. How (and if) magnetic dynamos depend on the strength of differential rotation in terms of $\alpha$ or on absolute shear $\Delta \Omega$ has not yet been empirically tested. Furthermore, different observing techniques measure different quantities, and authors express their results on stellar rotation laws in different variables. A variable frequently used is the lap time, i.e. the time it takes the equator to lap the polar regions (or vice versa), which is essentially the reciprocal of the shear $\Delta \Omega$. Differential rotation $\alpha=\Delta \Omega / \Omega$ is the shear divided by the angular velocity. Expressing rotation velocity in terms of rotation period $P$ essentially means the reciprocal of angular velocity $\Omega$, but using $\Delta P$ instead of $\Delta \Omega$ introduces several problems, since one has to consider whether $P$ denotes equatorial or polar rotation period, the latter being larger with solar-like differential rotation (cf. Reiners \& Schmitt 2003b). In this paper, I will express the rotation law in terms of $\alpha$ and $\Delta \Omega$, in order to search for correlations with rotation velocity. The quantity measured by the FTM is $\alpha$ with a fixed observational threshold of $\alpha_{\min } \approx 0.05$ thereby limiting the detection of deviations to rigid rotation. The uncertainty in $\alpha$ measured by FTM is approximately $\delta \alpha=0.1$. The perhaps more intuitive parameter for the physical consequences of differential rotation, however, is the shear $\Delta \Omega=\alpha \Omega$, where $\Omega$ has to be obtained from $v \sin i$ and the radius. When analyzing the rotation law in terms of $\Delta \Omega$, one has to keep in mind that it can never exceed angular velocity itself, if polar and equatorial regions are not allowed to rotate in opposite directions. In other words, differential rotation cannot be larger than $100 \%$.

\section{Observational data}

Data for this study have been compiled from observations carried out at different telescopes. Observations of field stars with projected rotation velocities higher than $v \sin i=45 \mathrm{~km} \mathrm{~s}^{-1}$ have been carried out with FEROS on the $1.5 \mathrm{~m}$ telescope $(R=48000)$, ESO, La Silla, or with FOCES $(R=40000)$ on the $2.2 \mathrm{~m}$ at CAHA. Slower rotating field stars were observed at higher resolution at the CES with the $3.6 \mathrm{~m}$ telescope, ESO, La Silla $(R=220000)$. Additionally, observations in open clusters were carried out with the multi-object facility FLAMES feeding VLT's optical high-resolution spectrograph UVES at a resolution of $R=47000$. Details of the spectra and instruments used are given in Table 1. Parts of the data were published in Reiners \& Schmitt (2003a,b) and Reiners et al. (2005). For more information about observations and data reduction, the reader is referred to these papers. Ten of the cluster targets (FLAMES/UVES), as well as 34 FOCES targets were not reported on in former publications ${ }^{1}$.

More than 600 stars were observed for this project during the last four years. For this analysis, I selected the 147 stars for which the ratio of the Fourier transform's zeros $q_{2} / q_{1}-$ the tracer of the rotation law - is measured with a precision better than 0.1 , i.e. better than $6 \%$ in case of the typical value of $q_{2} / q_{1}=1.76$. These 147 stars exhibit broadening functions that are (i) symmetric (to avoid contamination by starspots), and (ii) reveal rotation velocities between $v \sin i=12 \mathrm{~km} \mathrm{~s}^{-1}$ and $150 \mathrm{~km} \mathrm{~s}^{-1}$, the latter being an arbitrary threshold in order to minimize the amount of gravity-darkened stars with $v_{\text {eq }} \gg 200 \mathrm{~km} \mathrm{~s}^{-1}$ (Reiners 2003). Depending on the spectral

\footnotetext{
1 It should be noted that the FLAMES/UVES targets observed in open cluster fields are not necessarily cluster members, as was shown for example for Cl* IC 4665 V 102 in Reiners et al. (2005). A detailed investigation of cluster membership goes beyond the scope of this analysis; rotation velocity and spectral type make them sufficiently comparable to the (probable) field stars in this context.
} 
Table 1. Data used for this analysis. Spectra were taken with different high-resolution spectrographs. Detection of differential rotation requires higher resolution in slower rotators. The minimum rotation velocities required for determining the rotation law are given in Col. 4 . From the 147 spectra from which differential rotation could be determined, 28 stars show signatures of differential rotation.

\begin{tabular}{crcccc}
\hline \hline Instrument & Resolving power $\lambda / \Delta \lambda$ & Wavelength range $[\AA]$ & $\min . v \sin i\left[\mathrm{~km} \mathrm{~s}^{-1}\right]$ & Total \# of stars & \# of differential rotators \\
\hline CES & 220000 & 40 & 12 & 32 & 14 \\
FEROS & 48000 & 6000 & 45 & 23 & 3 \\
FOCES & 40000 & 6000 & 45 & 81 & 7 \\
FLAMES/UVES & 47000 & 2000 & 45 & 11 & 4 \\
\hline Total & & & 147 & 28 \\
\hline
\end{tabular}

resolution, the minimum rotation velocity is $v \sin i=12 \mathrm{~km} \mathrm{~s}^{-1}$ (CES) or $45 \mathrm{~km} \mathrm{~s}^{-1}$ (FEROS, FOCES, UVES, see also Table 1).

More than 450 stars have not been used in this analysis. Their broadening functions show a whole variety of broadening profiles. Many are slow rotators, although some were reported as rapid rotators in earlier catalogues (cf. Reiners \& Schmitt 2003b). A large number of spectroscopic binaries (or even triples) were found, and another large part of the sample shows spectra that are apparently distorted by starspots or other mechanisms that cause the spectra to appear asymmetric. For analyzing the rotation law from the broadening profile alone, these spectra are useless and are not considered in this work. They may be promising targets for DI techniques or other science; a catalogue of broadening profiles is in preparation.

Reiners \& Royer (2004) measured the rotation law in 78 stars of spectral type A including a few early F-type stars. This sample will only be incorporated in the HR-diagram in Sect. 6.1. As will be shown there, rotation laws in A-stars are fundamentally different from those in F-stars. For this reason, I will not incorporate the A-stars in the sample analyzed for rotation- and temperature-dependencies of differential rotation. One of the stars observed during this project has recently been studied with DI, with comparison of the results given in the appendix in Sect. A.

\section{Accuracy of rotation law measurements}

Projected rotation velocities $v \sin i$ were derived from the first zero $q_{1}$ in the Fourier transform as explained in Reiners \& Schmitt (2003b). As mentioned there, the precision of this measurement is usually $<1 \mathrm{~km} \mathrm{~s}^{-1}$, but simulations revealed a systematic uncertainty of $\sim 5 \%$ in $v \sin i$. Thus, I chose the maximum of the uncertainty in the intrinsic measurement and the $5 \%$ limit as the error on $v \sin i$.

The typical uncertainty for the determined values of relative differential rotation in terms of $\alpha=\Delta \Omega / \Omega$ is $\delta \alpha \approx 0.1$ (Reiners \& Schmitt 2003a). In slow rotators, this uncertainty is dominated by the noise level due to comparably sparse sampling of the profile even at very high resolution. In fast rotators, the profile (i.e., the zeros of the profile's Fourier transform) can be measured with very high precision. Here the uncertainty in $\alpha$ stems from poor knowledge of the limb darkening parameter $\epsilon$. For the case of a linear limb darkening law, the value of the measured ratio of the Fourier transform's first two zeros, $q_{1}$ and $q_{2}$, is in the range $1.72<q_{2} / q_{1}<1.85$
(Dravins et al. 1990). With the very conservative estimate that $\epsilon$ is essentially unknown $(0.0<\epsilon<1.0)$, every star with $1.72<q_{2} / q_{1}<1.85$ in this analysis was considered a rigid rotator, and $q_{2} / q_{1}<1.72$ was interpreted as solar-like differential rotation with the equator rotating faster than the polar regions. From the measured value of $q_{2} / q_{1}$, the parameter $\alpha / \sqrt{\sin i}$ was determined as explained above.

Stars can exhibit ratios of $q_{2} / q_{1}>1.85$ as well, but this value also does not agree with rigid rotation of a homogenous stellar surface. In the investigated sample of 147 stars, eight (5\%) exhibit a ratio of $q_{2} / q_{1}>1.85$. In contrast to solarlike differential rotation with the equator rotating more rapidly than polar regions, this case may be due to anti solar-like differential rotation with polar regions rotating faster than the equator. On the other hand, it can also be caused by a cool polar cap, which is expected to occur in rapidly rotating stars (Schrijver $\&$ Title 2001). The lower flux emerging from a cool polar cap makes the line center shallow and has the same signature as anti solar-like differential rotation. While anti solar-like differential rotation cannot be distinguished from a cool polar cap by investigating the rotation profile, the existence of a cool polar cap in rapidly rotating F-stars seems much more plausible than anti solar-like differential rotation. Differentiation between the two cases can only be achieved by measuring differential rotation independent of the line shape. For the scope of this paper, however, I will interpret stars with $q_{2} / q_{1}>1.85$ as rigid rotators with a cool polar cap.

\section{Rotation velocity and spectral type}

The observations carried out with FEROS, FOCES, UVES, and the CES are very homogeneous in terms of data quality and the investigated wavelength region, and only measurements that fulfill the criterion $\delta \alpha<0.1$ are considered. The targets have similar spectral types, but they do not form a statistically unbiased sample. The quality requirements discussed above make it difficult to analyze stars in a well-defined sample. This sample certainly is severely biased by observational and systematic effects. The most important bias is probably due to rotational braking, by connecting spectral type with rotational velocity in the presumably old field stars. Later spectral types suffer from stronger magnetic braking and are expected to be generally slower than earlier spectral types.

Projected rotation velocities $v \sin i$ of the sample stars are plotted versus color $B-V$ in Fig. 1, while differentially rotating 


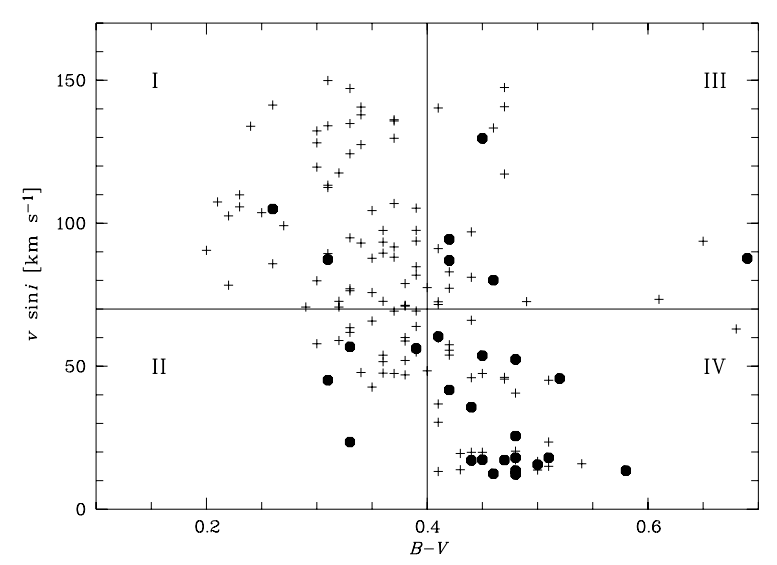

Fig. 1. Projected rotation velocity plotted vs. $B-V$ for the whole sample of 147 stars. Differential rotators are plotted as filled circles. Contents of subsamples I-IV are given in Table 2.

Table 2. Amount of differential rotators, total number of stars, and percentage of differential rotators for each subsample of Fig. 1.

\begin{tabular}{crrr}
\hline \hline Subsample & \# of diff. rotators & Total \# of stars & Percentage \\
\hline I & 2 & 57 & $4 \%$ \\
II & 4 & 24 & $17 \%$ \\
III & 5 & 21 & $24 \%$ \\
IV & 17 & 45 & $38 \%$ \\
\hline
\end{tabular}

stars (i.e. stars with $\alpha>0$ ) are indicated by filled circles and will be discussed in detail in Sect. 6. A clear dependence of $v \sin i$ on color is apparent, as expected. In Fig. 1, the sample is divided arbitrarily into four subsamples with projected rotation velocities that are higher (lower) than $v \sin i=70 \mathrm{~km} \mathrm{~s}^{-1}$, and color redder (bluer) than $B-V=0.4$. The total numbers of stars in each subsample and the numbers of differential rotators are given in Table 2. Most targets occupy regions I and IV. The scarcity in region II is due to the early F-type stars not being subject to strong magnetic braking, and since the sample mainly consists of field stars, most stars later than $B-V=0.4$ have been decelerated into region IV and do not appear in region III. Thus, slower rotating stars generally have a later spectral type in the sample. This implies that the effects of temperature and rotation velocity on the fraction of differentially rotating stars are degenerate in this sample; slow rotation implies late spectral type. It is therefore not possible to uniquely distinguish between the effects of rotation and spectral type in the investigated sample. This degeneracy has to be kept in mind when trying to interpret rotation- and temperaturedependencies of differential rotation in the following chapters.

\section{Results}

As a first result of the profile analysis, I present $v \sin i$ and the measurement of the rotation law for all 147 objects in Appendix B. Fields stars (FEROS, FOCES, and CES observations) are compiled in Table B.1, FLAMES/UVES targets in Table B.2.
In the sample of 147 stars for which the rotation law was measured, $28(19 \%)$ exhibit signatures of solar-like differential rotation $\left(q_{2} / q_{1}<1.72\right)$. CES-, FEROS-, and FOCESsamples contain field stars of spectral type later than F0, with the majority of them brighter than $V=6$ mag. Stars in fields of open clusters contained in the UVES-sample probably are mostly younger than the field stars, and all of them should have reached the main sequence (Stahler \& Palla 2004).

\subsection{Differential rotation in the HR-diagram}

All currently available measurements of the stellar rotation law from rotation profile shape (i.e., 147 stars from this sample and 78 stars from Reiners \& Royer 2004) are plotted in an HR-diagram in Fig. 2. For the field and A-stars, effective temperature $T_{\text {eff }}$ and bolometric magnitude $M_{\text {bol }}$ were derived from uvby $\beta$ photometry taken from Hauck \& Mermilliod (1998) using the program UVBYBETA published by Moon (1985). For $T_{\text {eff }}$ a new calibration by Napiwotzki et al. (1993) based on the grids of Moon \& Dworetsky (1985) was used, and the statistical error of the temperature determination is about $\Delta T_{\mathrm{eff}}=150 \mathrm{~K}$. For three of the field stars, no $u v b y \beta$ photometry is available; all three are rigid rotators, so no value of $T_{\mathrm{eff}}$ was calculated to avoid inconsistencies. For the cluster stars, no $u v b y \beta$ data is available, so radius and temperature are estimated from $B-V$ color using zero-age main sequence (ZAMS) polynomials taken from Gray (1976), i.e., they are assumed to be young. For them, $M_{\mathrm{bol}}$ is calculated from

$M_{\text {bol }}=42.36-10 \log T_{\text {eff }}-5 \log R / R_{\odot}$,

with $R_{\odot}$ the solar radius. To get absolute $V$-magnitudes $M_{V}$ from bolometric magnitudes $M_{\mathrm{bol}}$, the bolometric correction calibrated from Hayes (1978) was applied.

In Fig. 2, stars are plotted as open or filled circles that indicate rigid $(\alpha=0)$ or differential rotation $(\alpha>0)$, respectively. Circle sizes display projected rotation velocities $v \sin i$ as explained in the figure. The 147 stars given in Tables B.1 and B.2 are plotted as black symbols, while stars from Reiners \& Royer (2004) are plotted as grey symbols. Evolutionary main sequence tracks for $M=1.2,1.4,1.6,2.0$, and $3.0 M_{\odot}$ and the ZAMS from Siess et al. (2000) are also shown. Near the interface of A-stars and F-stars, the "granulation boundary" from Gray \& Nagel (1989) is indicated with dashed lines. This is the region where line bisectors measured in slow rotators show a reversal. For dwarfs and subgiants, the "granulation boundary" coincides with theoretical calculations of the "convection boundary" and thus can be identified as the region where deep envelope convection disappears (cf. Gray \& Nagel 1989). The exact region of this boundary is not defined well and may depend on factors other than temperature and luminosity.

The stars shown in Fig. 2 cover a wide range in temperature on both sides of the convection boundary. Because field stars are investigated, many stars have evolved away from the ZAMS. Rotation velocity as indicated by symbol size follows the well-known behavior of magnetic braking; late type stars are generally slowed down and exhibit slower rotation velocities. The striking fact visible in Fig. 2 is that none of the differential rotators detected with FTM lies significantly on the hot 


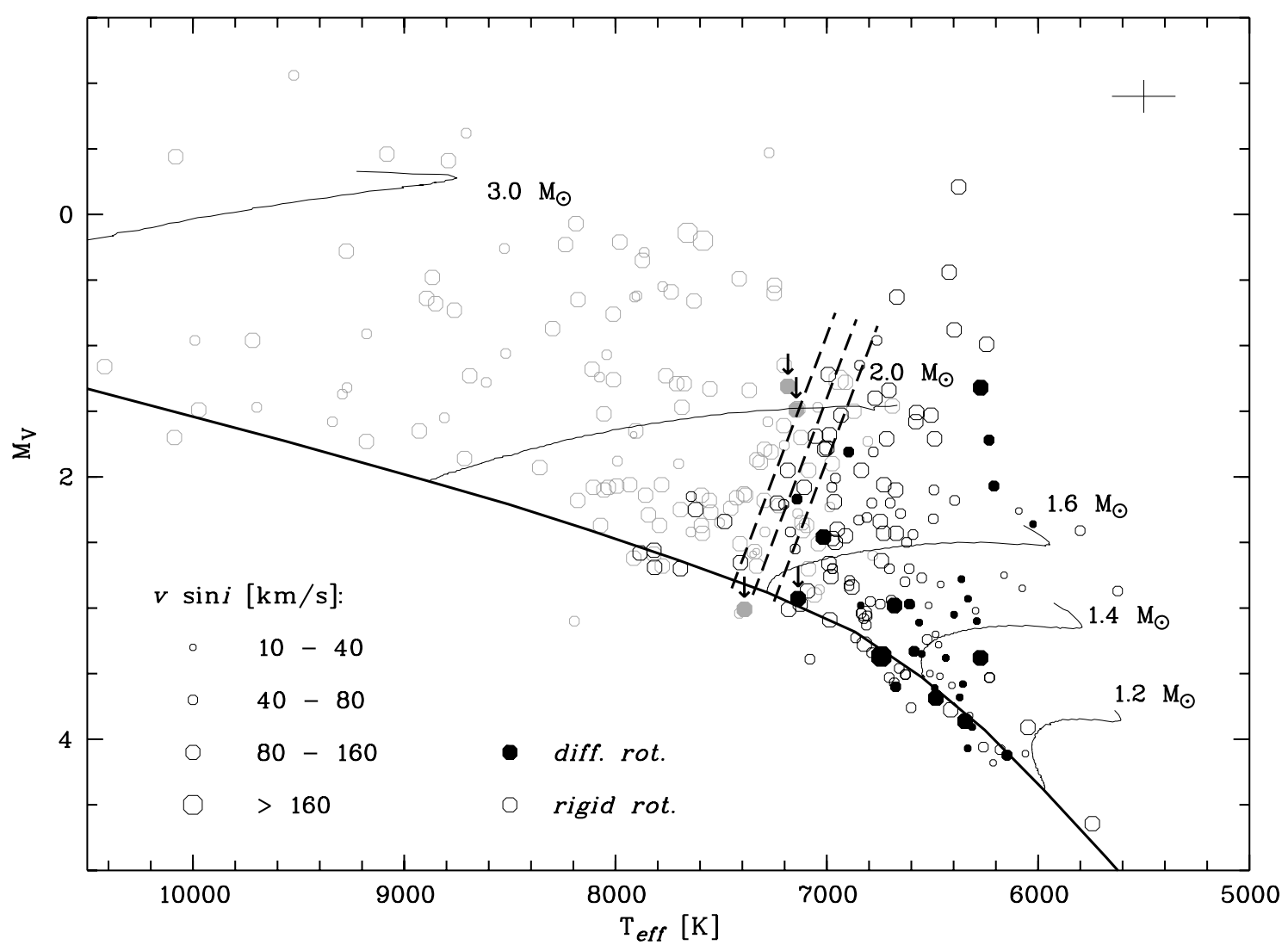

Fig. 2. HR-diagram of all currently available measurements of latitudinal differential rotation from FTM. Stars analyzed in this work are plotted in black, and stars from the A-star sample in Reiners \& Royer (2004) in grey. Differential rotators are indicated by filled circles. Symbol size represents ranges of projected rotation velocity $v \sin i$ as explained in the figure. A typical error bar is given in the upper right corner. Evolutionary tracks and the ZAMS from Siess et al. (2000) are overplotted. Dashed lines mark the area of the granulation boundary according to Gray \& Nagel (1989), while no deep convection zones are expected on the hot side of the boundary. Downward arrows indicate the four stars from Table 3 (see text).

side of the convection boundary - latitudinal differential rotation has only been detected in stars believed to possess a deep convective envelope.

The largest group of differential rotators can be found near the ZAMS at all temperatures that are cooler than the convection boundary. The convection boundary itself is also populated by differential rotators. A few others can be found far away from the ZAMS (at masses $\gtrsim 1.6 M_{\odot}$ ) to the right end of the main sequence tracks. There is a hint that differential rotators are lacking in the region between these few rotators and those near the ZAMS and again between them and those near the convection boundary, although this region is occupied by rigidly rotating stars (i.e., stars with differential rotation weaker than the observational threshold). However, it is not clear from the available sample whether temperature (and evolutionary stage) or rotation velocity may be the important parameter in determining differential rotation in F-type stars (see Sect. 5).

\subsection{Differentially rotating A-stars at the convection boundary}

Among all the differentially rotating stars shown as filled circles in Fig. 2, the four stars with the strongest shear $\Delta \Omega$ are found very close to the convection boundary. This group of
Table 3. Four stars with extreme values of differential rotation $\Delta \Omega$. It is speculated that these objects represent a special class of targets (marked with downward arrows in Fig. 2) in a narrow region of rotation velocity and effective temperatures near the convection boundary.

\begin{tabular}{lrcrccc}
\hline \hline \multicolumn{2}{c}{ Star } & Sp & $\begin{array}{c}v \sin i \\
{\left[\mathrm{~km} \mathrm{~s}^{-1}\right]}\end{array}$ & $\begin{array}{c}\Delta \Omega \sin i \\
{\left[\mathrm{rad} \mathrm{d}^{-1}\right]}\end{array}$ & $\begin{array}{c}T_{\text {eff }} \\
{[\mathrm{K}]}\end{array}$ & $M_{V}$ \\
\hline HD & 6869 & A9 & $95 \pm 5^{1}$ & $2.3 \pm 1.5^{1}$ & 7390 & 3.01 \\
HD & 60555 & A6 & $109 \pm 5^{1}$ & $1.3 \pm 0.7^{1}$ & 7145 & 1.49 \\
HD & 109238 & F0 & $103 \pm 4^{1}$ & $1.3 \pm 0.6^{1}$ & 7184 & 1.31 \\
IC 4665 V 102 & A9 & $105 \pm 12^{2}$ & $3.6 \pm 0.8^{2}$ & 7136 & 2.93 \\
\hline
\end{tabular}

${ }^{1}$ Reiners \& Royer (2004); ${ }^{2}$ Reiners et al. (2005).

rapidly rotating late A-/early F-type stars is listed in Table $3^{2}$. All four exhibit very similar effective temperatures around $T_{\text {eff }}=7200 \mathrm{~K}$, putting them into the region where convective

\footnotetext{
${ }^{2}$ It should be noted that the only star in this group, that lies on the cool side of the convection boundary, is $\mathrm{Cl}^{*}$ IC $4665 \mathrm{~V} 102$. Temperature and luminosity were estimated from $B-V$ color using

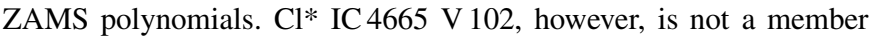
of the cluster IC 4665 (Reiners et al. 2005), hence its position in the HR-diagram is most uncertain. It may have a much higher absolute luminosity that shifts it even closer towards the convection boundary.
} 
envelopes are extremely shallow. The four stars show remarkably similar projected rotation velocities all within $10 \%$ of $v \sin i=100 \mathrm{~km} \mathrm{~s}^{-1}$, and all four have rotation periods shorter than $P=2 \mathrm{~d}$. Two of them, HD 6869 and $\mathrm{Cl}^{*} \mathrm{IC} 4665 \mathrm{~V} \mathrm{102}$, exhibit a shear that is as strong as $\Delta \Omega>2 \mathrm{rad} \mathrm{d}^{-1}$ at rotation periods that are shorter than one day. This contrasts the 34 other stars that show comparably small rotation periods but have different temperatures or surface velocities. None of the 34 other rapid rotators exhibits a shear $\Delta \Omega \sin i$ in excess of $0.7 \mathrm{rad} \mathrm{d}^{-1}$, i.e. a factor of three weaker than the two mentioned above, and only three of the 34 show $\Delta \Omega>0$ at all.

The fact that the four strongest differential rotators are found at hot temperatures and high rotation velocities contradicts the general trend that differential rotation is more common in slowly rotating cool stars, which will be discussed in the following sections. All four are very close to the convection boundary or even on its blue side, meaning extremely shallow convective envelopes. This leads to the suspicion that the mechanism responsible for the strong shear is different from the one that drives the shear in stars with deeper convection zones. This is supported by the observation that all four stars also exhibit very similar (and comparably large) surface velocities of about $100 \mathrm{~km} \mathrm{~s}^{-1}$. It is thus suggested that the strong surface shear in the four stars in Table 3 is not comparable to the rest of the sample, but is facilitated by the high surface velocity in a particularly shallow convective envelope. Such a mechanism could be supported by eigenmodes that are comparable to pulsational instabilities, for example in $\delta$ Scu stars, but further investigation is beyond the scope of this paper.

\subsection{The fractional amount of differential rotators}

In this section, the hypothesis that the fraction of differentially rotating stars with $\alpha>0$ is independent of rotation velocity $v \sin i$ and color $B-V$ will be tested. The actual strength of differential rotation is not taken into account, but will be investigated in the following sections.

In Fig. 1, the whole sample of 147 stars has been divided into the four arbitrary regions mentioned in Sect. 5 (dividing at $v \sin i=70 \mathrm{~km} \mathrm{~s}^{-1}$ and $B-V=0.4$ ). The number of stars and differential rotators $(\alpha>0$, for this sample this means stars with $\alpha$ above the observational threshold of $\alpha_{\min } \approx 0.05$ ), and the percentage of differential rotators is given in Table 2 . The percentage of differentially rotating stars among slow rotators in regions II and IV is higher than it is among rapid rotators in regions I and III, respectively; a trend towards a higher fraction of differential rotators at slower rotation velocity is visible in both color regimes. The same is true for the percentage of differentially rotating stars among late-type stars in regions III and IV. A comparison to earlier type stars among regions I and II yields a higher fraction of differentially rotating stars towards later spectral type. I tested the hypothesis that subsamples I-IV are drawn from the same distribution with a total mean of $19 \%$ differential rotators. Samples II and III are consistent with this hypothesis (5 and 4 expected differential rotators, respectively). For samples I and IV, the hypothesis can be rejected at a $99 \%$ level $(99.3 \%, 11$ expected, and $99.6 \%$,
8 expected for samples I and IV, respectively). Thus, the fraction of differential rotators with $\alpha$ larger than the observational threshold of $\alpha_{\min } \approx 0.05$ - which does not depend on $v \sin i$ or color - is not constant. It is larger in slower rotators and stars of a later spectral type.

The distribution of differential rotators in $v \sin i$ and $B-V$ is investigated further in Fig. 3. The upper panel shows the total number of stars divided into five bins in $B-V$ (left), and seven bins in $v \sin i$ (right). The lower panel of Fig. 3 displays the fraction of differential rotators $(\alpha \gtrsim 0.05)$ in the respective color/rotation bins with $2 \sigma$-errors. For example, $48 \%$ of the 25 stars with projected rotation velocities $v \sin i$ between 10 and $30 \mathrm{~km} \mathrm{~s}^{-1}$ show signatures of differential rotation. Although some bins are sparsely populated and have large errors, the change from color $B-V=0.4$ to $B-V=0.5$ and the transition from slow rotators to stars with $v \sin i>30 \mathrm{~km} \mathrm{~s}^{-1}$ are significant. The trends indicated in Table 2 stand out in the lower panel of Fig. 3. In this sample, profiles with $\alpha \gtrsim 0.05$ are more frequent in slow rotators, which implies that they are more frequent in stars of later spectral type (due to the sample bias, cf. Sect. 5).

\subsection{Differential rotation and rotation period}

In this and the following sections, I will investigate the strength of differential rotation and especially the maximum strength of differential rotation at different rotation rates. I discuss differential rotation in terms of $\alpha=\Delta \Omega / \Omega$ in Sect. 6.4.1, and analyze the shear $\Delta \Omega$ in Sect. 6.4.2. Although both quantities essentially have the same meaning, it is instructive to look at both of them separately. Rotation speed will be expressed as a function of equatorial rotation period instead of rotation velocity. The rotation period itself is not measured for the majority of stars, so I will use $P / \sin i$ instead, as calculated from measured $v \sin i$ and the radius according to Eq. (3).

In the left and right panels of Fig. 4, values of differential rotation $\alpha$ and absolute shear $\Delta \Omega$ are plotted against projected rotation period $P / \sin i$, respectively. From the 147 stars in Table 1 , only the 28 stars with signatures of differential rotation are shown, while the other 119 objects populate the $\alpha=\Delta \Omega / \Omega=0$ region at $0.5 \mathrm{~d}<P / \sin i<11.0 \mathrm{~d}$. Field stars from this sample with available $u v b y \beta$ measurements are plotted as filled circles, while the four cluster targets for which the ZAMS-age has been assumed are plotted as open circles. In both panels, the three differentially rotating stars from Reiners \& Royer (2004), which have been discussed in Sect. 6.2, are marked as crosses.

In the left and right panels of Fig. 4, the long dashed lines qualitatively indicate the upper envelope of $\alpha / \sqrt{\sin i}$ and $\Delta \Omega \sqrt{\sin i}$, respectively. No fit was attempted, so the lines should only guide the eye to clarify what will be discussed in the next sections.

\subsubsection{Differential rotation $\alpha$}

The advantage of using $\alpha$ is that it is measured directly and its detection does not depend on rotation period, hence radius, 

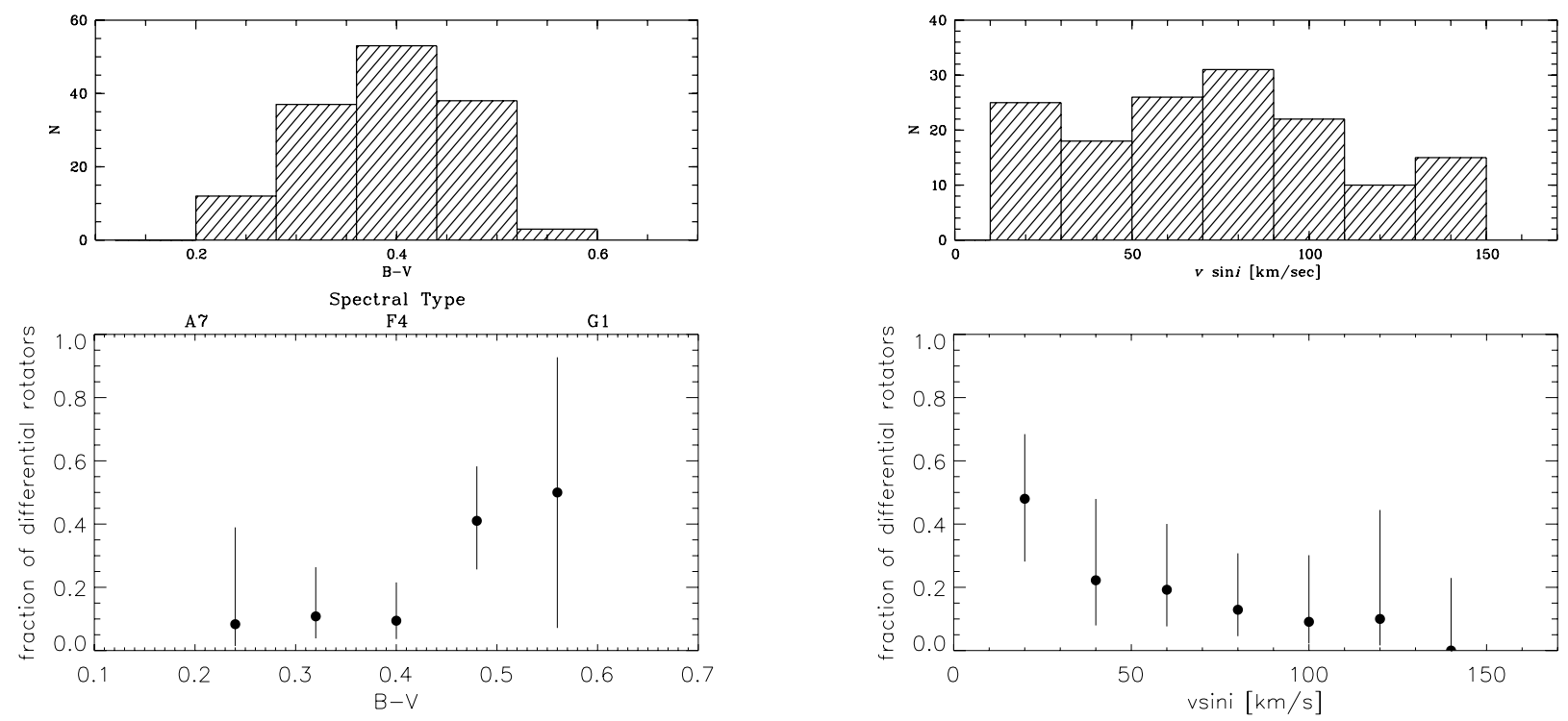

Fig. 3. Upper panel: histograms of the sample in $B-V$ (left column) and $v \sin i$ (right column). Lower panel: fraction of stars with strong relative differential rotation in the bins plotted in the upper panel. $2 \sigma$-errors are overplotted.
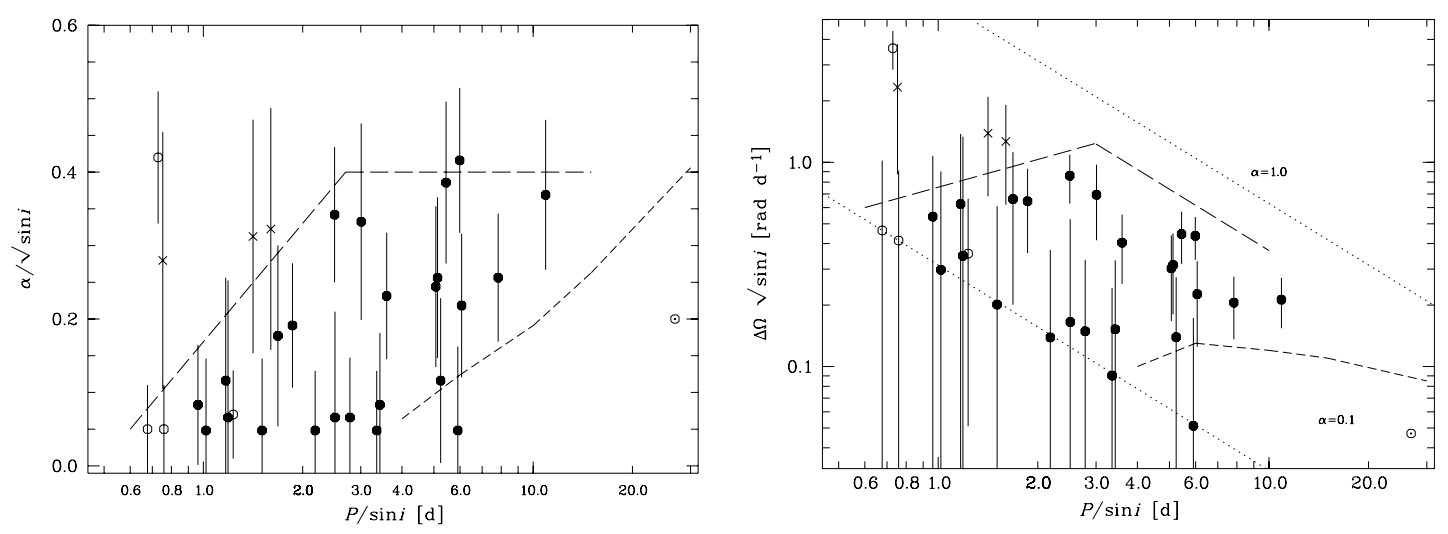

Fig. 4. Differential rotation vs. projected rotation period $P / \sin i$. Left: differential rotation $\alpha=\Delta \Omega / \Omega$; right: absolute shear $\Delta \Omega$ (note the logarithmic scale on the ordinate). Field F-stars are indicated by filled circles, open circles display stars from cluster observations. Crosses mark the three differential rotators from Reiners \& Royer (2004). Short dashed lines are models from Küker \& Rüdiger (2005) for an F8-star with a viscosity parameter of $c_{v}=0.15$. The four stars with extraordinarily strong shear $\Delta \Omega$ were discussed in Sect. 3, a different mechanism is suggested to cause their strong shear. For the remaining stars, the upper envelopes are indicated qualitatively by the long dashed lines. In the right panel, dotted lines show regions of constant differential rotation $\alpha=0.1$ (approximate sensitivity of FTM) and $\alpha=1.0$ (maximum if no counter-rotation of the polar regions is allowed for).

while measuring $\Delta \Omega$ does. Differential rotation $\alpha$ is smaller than 0.45 for all rotation periods. While a minimum threshold of $(\alpha / \sqrt{\sin i})_{\min } \approx 0.05$ applies, the observational technique has no limitations towards high values of $\alpha$. Thus, the highest detected value of differential rotation $(\alpha / \sqrt{\sin i})_{\max } \approx 0.45$ is not due to limitations of the FTM.

At rotation periods between two and ten days, the targets populate the whole region $0<\alpha / \sqrt{\sin i}<0.45$, while the slower targets could not be analyzed due to the limitations of the FTM (cf. Sect. 2). Among the rapid rotators with projected rotation periods that are shorter than two days, the upper envelope shows a clear decline among the F-stars (filled circles in the left panel of Fig. 4). Except for the group of A-stars discussed in Sect. 6.2 (listed in Table 3), no star with a projected rotation period less than two days shows $\alpha>0.2$, and no star with $P / \sin i<1$ d shows $\alpha>0.1$. Neglecting those four stars, the maximum value in differential rotation, $(\alpha / \sqrt{\sin i})_{\max }$, grows from virtually zero at $P=0.5 \mathrm{~d}$ to $(\alpha / \sqrt{\sin i})_{\max } \approx 0.45$ in stars slower than $P=2 \mathrm{~d}$. In slower rotators, $(\alpha / \sqrt{\sin i})_{\max }$ remains approximately constant.

\subsubsection{Absolute shear $\Delta \Omega$}

Absolute shear $(\Delta \Omega \sqrt{\sin i})$ is shown in the right panel of Fig. 4 . Since observed values of both $\alpha$ and $\Omega$ depend on inclination $i$, the observed absolute shear is

$\Delta \Omega_{\mathrm{obs}}=\alpha_{\mathrm{obs}} \Omega_{\mathrm{obs}}=\alpha / \sqrt{\sin i} \Omega \sin i=\Delta \Omega \sqrt{\sin i}$.

In this and the following sections, I omit the factor $\sqrt{\sin i}$ for readability. Note that in the case of small inclination angles, 
the value of $\Delta \Omega$ can be larger than $\Delta \Omega \sqrt{\sin i}$, while $\alpha$ can be smaller than $\alpha / \sqrt{\sin i}$. In the right panel of Fig. 4, the two dotted lines indicate the slopes of constant differential rotation $\alpha=\Delta \Omega / \Omega$. The upper line is for $\alpha=1.0$, i.e., the maximum differential rotation possible regardless of the observation technique used, and the lower line shows $\alpha=0.05$, the approximate minimum threshold for the FTM, as explained above.

As expected from Sect. 6.4.1, the F-stars form a relatively smooth upper envelope in the maximum absolute shear $\Delta \Omega_{\max }$, as observed at different rotation rates. The slowest rotators exhibit low values of $\Delta \Omega_{\max } \approx 0.2 \mathrm{rad} \mathrm{d}^{-1}$ at $P \approx 10 \mathrm{~d}$. $\Delta \Omega_{\max }$ grows towards a faster rotation rate with a maximum between two and three days before it diminishes slightly with more rapid rotation. The strongest differential rotation occurs at rotation periods $P$ between two and three days at a magnitude of $\Delta \Omega \approx 1 \mathrm{rad} \mathrm{d}^{-1}$ (i.e., lapping times on the order of $10 \mathrm{~d}$ ). At higher rotation rates in the range $0.5 \mathrm{~d}<P<2 \mathrm{~d}$, the maximum shear has a slope of roughly $\Delta \Omega \propto P^{+0.4}$. This slope, however, is not constrained well due to the large uncertainties and sparse sampling. The data are also consistent with a plateau at $\Delta \Omega \approx 0.7 \mathrm{rad} \mathrm{d}^{-1}$ for $0.5<P<3 \mathrm{~d}$. At slower rotation, right from the maximum, the slope is approximately $\Delta \Omega \propto P^{-1}$.

Küker \& Rüdiger (2005) recently calculated $\Delta \Omega$ in an F8 star for different rotation periods. Their results for $\Delta \Omega(P)$ (with a viscosity coefficient $c_{v}=0.15$ ) are displayed qualitatively in both panels of Fig. 4 as a short-dashed line (Fig. 6 in Küker \& Rüdiger 2005). One of their results is that in their model $\Delta \Omega$ does not follow a single scaling relation for all periods (as was approximately the case in the calculations by Kitchatinov \& Rüdiger 1999), but that a maximum shear arises at a rotation period of about $P=7 \mathrm{~d}$ in the case of the modeled F8-star. Comparison of their calculations (right panel of Fig. 4) to the upper envelope suggested in this work (longdashed line) still shows a large quantitative discrepancy. The qualitative slopes of both curves, however, are in reasonable agreement with each other. The theoretical curve was calculated for an F8-star. Küker \& Rüdiger (2005) also show $\Delta \Omega(P)$ for a solar-type star, where $\Delta \Omega(P)$ is essentially moved towards higher rotation periods and lesser shear; i.e. the shortdashed curve in the right panel of Fig. 4 moves to the lower right for later spectral types. Although earlier spectral types are not calculated by Küker \& Rüdiger (2005), it can be expected that $\Delta \Omega(P)$ will shift towards higher shear and shorter rotation periods in stars of an even earlier spectral type. Since most stars investigated in this sample are earlier than F8, this would suggest qualitative consistency between theoretical curves and the slope of $\Delta \Omega_{\max }$ shown here.

\subsubsection{Comparing different techniques}

Differential rotation measurements are now available from a variety of observational techniques (see Sect. 1), comparison of results from techniques becomes possible. However, such a comparison has to be carried out with great care. Photometrically measured periods, for example, are only sensitive to latitudes covered by spots; they reflect only parts of the rotation law and are always lower limits. Furthermore, temperature has been shown to be the dominating factor for the strength of differential rotation (Kitchatinov \& Rüdiger 1999; Küker \& Rüdiger 2005; Barnes et al. 2005), which has to be taken into account when analyzing the rotation dependence of differential rotation.

In the past, analyses of relations between rotation and differential rotation have generally assumed a monotonic scaling relation between period $P$ (or angular velocity $\Omega=2 \pi / P$ ) and $\Delta \Omega$. Such a relation was expected from calculations by Kitchatinov \& Rüdiger (1999). As mentioned above, Küker \& Rüdiger (2005) recently presented new calculations showing that the $\Delta \Omega$ vs. $\Omega$-relation may have a temperature dependent maximum.

Searching for dependence on angular velocity, Barnes et al. (2005) recently compiled data from differential rotation measurements from DI, photometric monitoring, and FTM. Fitting a single power law to the compiled data, they derive $\Delta \Omega \propto$ $\Omega^{+0.15 \pm 0.10}$, which is compared to the case of a G2 dwarf calculated in Kitchatinov \& Rüdiger (1999). From the latter, they cite the theoretical G2 dwarf relation as $\Delta \Omega \propto \Omega^{+0.15}$, and claim agreement to their fit. Although the work of Kitchatinov \& Rüdiger (1999) has been superseded by Küker \& Rüdiger (2005), it should be mentioned that Kitchatinov \& Rüdiger (1999) report $\Delta \Omega \propto \Omega^{-0.15}$, implying stronger shear for slower rotation instead of weaker (note that Küker \& Rüdiger 2005, also report a negative exponent for their solar-like star model at periods less than $20 \mathrm{~d}$ ). In fact, the large scatter in the compilation of all measurements from different techniques (Fig. 3 in Barnes et al. 2005) and the severe bias due to systematic uncertainties (like the lack of small values $\Delta \Omega$ in the sample measured with FTM) leads to any conclusion about the period dependence from such a heterogeneous sample very uncertain. The new data in this paper does not significantly improve this situation and no analysis that improves upon the one performed by Barnes et al. (2005) can be expected.

Although the constantly growing amount of differential rotation measurements provides a relatively large sample, the results from analyzing all measurements as one sample do not yet provide convincing evidence for a unique rotation dependence of differential rotation over the whole range of rotation periods.

\subsection{Upper bound of differential rotation and effective temperature}

Effective temperature is the second parameter after rotation to govern stellar differential rotation, since convection zone depth, as well as convection velocity, are very sensitive to $T_{\text {eff }}$. Küker \& Rüdiger (2005) and Kitchatinov \& Rüdiger (1999) report stronger differential rotation with higher effective temperatures when comparing a solar-type star to stars of spectral types F8 and K5. The differential rotators in the sample of F-stars investigated here span a range in effective temperature between $6000 \mathrm{~K}$ and $7150 \mathrm{~K}$. As a result, analysis of temperature effects on differential rotation is limited by the small range of targets in $T_{\text {eff }}$, and is biased by the large range in rotation periods, as discussed above. Thus, I limit the analysis of 


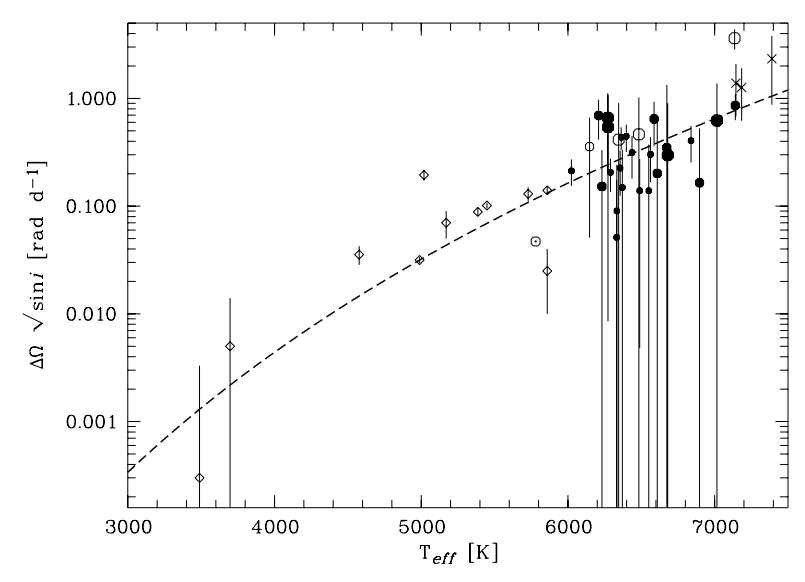

Fig. 5. Differential rotation $\Delta \Omega$ with effective temperature $T_{\text {eff }}$ for sample stars (symbols as in Fig. 4). Big circles indicate large rotation velocity. Stars analyzed with DI are indicated as squares, and the fit to DI targets from Barnes et al. (2005) is overplotted. F-stars with the strongest differential rotation are consistent with the fit.

temperature dependence to a comparison to differential rotation in stars that are significantly cooler than $T_{\text {eff }}=6000 \mathrm{~K}$.

Considering stars with effective temperatures in the range $3400 \mathrm{~K}<T_{\text {eff }}<6000 \mathrm{~K}$, Barnes et al. (2005) found a powerlaw dependence in their measurements of absolute shear $\Delta \Omega$ on stellar surface temperature. Their result is compared to the sample of this work in Fig. 5. Absolute shear follows the trend expected by Küker \& Rüdiger (2005) and Kitchatinov \& Rüdiger (1999) with a stronger surface shear at higher effective temperatures. Although a large scatter is observed in the F-star measurements, they qualitatively follow this trend and connect to cooler stars at roughly the expected values. In addition, the rotation rate of the F-stars is indicated by symbol size, larger symbols displaying higher rotation rate. Stars exhibiting a shear in excess of the expected rate for their temperature tend to show very rapid rotation. Thus, the investigated F-stars agree qualitatively with the temperature fit derived by Barnes et al. (2005). A more quantitative analysis is complicated by the large scatter among measurements of absolute shear, which is visible also in the sample of Barnes et al. (2005). Again, it should be noted that the values plotted for the F-star sample only display stars for which signatures of differential rotation have been measured. A large number of stars populate the region of weaker surface shear or rigid rotation $(\alpha=\Delta \Omega=0)$, and the temperature law applies only to the strongest differential rotators.

\section{Conclusions}

The sample of stars with rotation laws measured from spectral broadening profiles with the FTM is constantly growing. In this work, 44 new observations of stars of spectral type $F$ and later were added to the results from former publications. Currently, rotation laws have been analyzed in a homogeneous data set of 147 stars of spectral type F and later, and in a second data set in 78 stars of spectral type A. Among all these observations covering the temperature range between $5600 \mathrm{~K}$ and $10000 \mathrm{~K}$ (including A-stars from Reiners \& Royer 2004), 31 stars exhibit signatures of solar-like differential rotation. Only three of them are of spectral type A. In the HR-diagram, differential rotators appear near and on the cool side of the convection boundary. No differentially rotating star hotter than $7400 \mathrm{~K}$ is known, and it is obvious that the signatures of solar-like differential rotation are closely connected to the existence of deep convective envelopes.

Most differential rotators can be found near to the ZAMS at young ages, but due to the limited sample and severe selection effects, this needs confirmation from a less biased sample. Particularly, a number of slower rotators with temperatures around $T_{\text {eff }}=6600 \mathrm{~K}$ are needed.

Four differential rotators are very close to the convection boundary. All four show extraordinarily strong absolute shear and exhibit projected rotation velocities within $10 \%$ around $v \sin i=100 \mathrm{~km} \mathrm{~s}^{-1}$. It is suggested that these stars form a group of objects in which rotation velocity and convection zone depth facilitate very strong absolute shear and that the mechanism causing the shear is different from the later F-type stars.

Among the F-stars, differential rotation occurs in the whole range of temperatures and rotation rates. The sample of 147 stars of spectral type $\mathrm{F}$ and later was investigated for the dependence of differential rotation on rotation and temperature. 28 of them (19\%) exhibit signatures of differential rotation. The distribution of differential rotation was approached with two different strategies: (i) investigation of the fraction of stars exhibiting differential rotation $(\alpha>0)$; and (ii) analysis of the maximum $\alpha$ and maximum $\Delta \Omega$ as a function of rotation period and temperature. The first approach reflects the typical values of $\langle\alpha\rangle$ and $\langle\Delta \Omega\rangle$ at any given temperature and rotation rate, while the second focuses on the question how strong the absolute shear can possibly be in such stars. Due to the large uncertainties in the measurements and the high minimum threshold in differential rotation, the mean values of $\alpha$ and $\Delta \Omega$ are not particularly meaningful. Furthermore, it is not clear whether a smooth transition from stars exhibiting strong differential rotation to "rigidly" rotating stars $(\alpha<0.05)$ or a distinction between these two groups exists, and the mentioned approaches were preferred for the analysis.

In the sample, hotter stars generally rotate more rapidly, and effects due to rotation velocity and temperature cannot be disentangled. The distribution of differential rotators depends on color and/or rotation rate, and the fraction of stars with differential rotation $(\alpha>0)$ increases with cooler temperature and/or slower rotation. It is not clear what this means to the mean shear, $\langle\Delta \Omega\rangle$. For example, it is not inconsistent with $\langle\Delta \Omega\rangle$ being constant at all rotation rates. In this case, $\langle\alpha\rangle=\langle\Delta \Omega / \Omega\rangle$ would be smaller in more rapidly rotating stars, and thus a lower fraction of stars would exhibit differential rotation $\alpha$ above the observational threshold, as is observed.

On the other hand, the maximum observed values of differential rotation, $\alpha_{\max }$, and of the absolute shear, $\Delta \Omega_{\max }$, do vary depending on the rotation rate. The strongest absolute shear of $\Delta \Omega \approx 1 \mathrm{rad} \mathrm{d}^{-1}$ is found at rotation periods between two and three days with significantly smaller values in slower rotators. The more rapidly rotating stars show a slight decrease in absolute shear as well, although the sparse data are also consistent with a plateau at $\Delta \Omega \approx 0.7 \mathrm{rad} \mathrm{d}^{-1}$ for $0.5 \mathrm{~d}<P<3 \mathrm{~d}$. A maximum in differential rotation $\Delta \Omega$ has recently been predicted 
by Küker \& Rüdiger (2005) for an F8-star, although of lesser strength and at a slower rotation rate, a difference that may in parts be due to their later spectral type.

The investigated sample does not cover a wide range in effective temperature since only very few late-type field stars rotate fast enough for the method applied. Although temperature is expected to strongly influence the strength of differential rotation, the large range in rotation rate and the connection between rotation rate and temperature in the sample makes conclusions about temperature effects insecure. The results were compared to differential rotation measurements in cooler stars and found in qualitative agreement with an extrapolation of the empirical temperature dependence that Barnes et al. (2005) found when analyzing a sample of differentially rotation measurements done with DI.

The implications of the relations discussed here for stellar magnetic activity and the nature of the dynamo working in F-type stars still remain unclear from an observational point of view. Naively, one would expect stronger magnetic activity to occur in stars with stronger differential rotation among groups of comparable temperature or rotation rate. Those stars for which X-ray measurements are available, however, do not yet exhibit such a trend, but a meaningful investigation is hampered by the limited amount of data points available (especially for comparable temperature or rotation velocity). It has been shown that qualitative conclusions can be derived from the currently available measurements of stellar rotation laws, but a more detailed investigation of the consequences on the dynamo operating in F-type stars has to wait until a statistically betterdefined sample of stars is available.

Acknowledgements. I am thankful to G. Basri for carefully reading the manuscript and for very helpful discussions, and to J. Schmitt for valuable comments on an earlier version of the manuscript. I thank the referee, Dr. John Barnes, for a careful and very constructive report. A.R. has received research funding from the European Commission's Sixth Framework Programme as an Outgoing International Fellow (MOIF-CT-2004-002544).

\section{References}

Balthasar, H., Vázques, M., \& Wöhl, H. 1986, A\&A, 185, 87

Barnes, J. R., Collier Cameron, A., Donati, J.-F., et al. 2005, MNRAS, 357, L1
Bruning, D. H. 1981, ApJ, 248, 271

Domiciano de Souza, A., Zorec, J., Jankov, S., et al. 2004, A\&A, 418, 781

Donahue, R. A., Saar, S. H., \& Baliunas, S. L. 1996, ApJ, 466, 384

Donati, J.-F., \& Collier Cameron, A. 1997, MNRAS, 291, 1

Dravins, D., Lindegren, L., \& Torkelsson, U. 1990, A\&A, 237, 137

Gizon, L., \& Solanki, S. K. 2004, Sol. Phys., 220, 169

Gray, D. F. 1976, The observation and analysis of stellar photospheres (New York: Wiley)

Gray, D. F. 1977, ApJ, 211, 198

Gray, D. F. 1984, ApJ, 277, 640

Gray, D. F. 1998, 10th Cambridge Workshop on Cool Stars, Stellar Systems and the Sun, ASP Conf. Ser., 154

Gray, D. F., \& Nagel, T. 1989, ApJ, 341, 421

Hall, D. S. 1991, in The Sun and the cool Stars (New York: Springer Verlag)

Hayes, D. S. 1978, IAU Symp., 80, ed. A. G. D. Philip, \& D. S. Hayes, 65

Hauck, B., \& Mermilliod, M. 1998, A\&AS, 129, 431

Johns-Krull, C. M., \& Valenti, J. A. 1996, ApJ, 459, 95

Kitchatinov, L. L., \& Rüdiger, G. 1999, A\&A, 344, 911

Küker, M., \& Rüdiger, G. 2005, A\&A, 422, 1023

Marcy, G. W. 1984, ApJ, 276, 286

Marsden, S. C., Waite, I. A., Carter, B. D., \& Donati, J.-F. 2005, MNRAS, 359, 711

Moon, T. T. 1985, Coom. Univ. London Obs., 78

Moon, T. T., \& Dworetsky, M. M. 1985, MNRAS, 217, 305

Napiwotzki, R., Schönberner, D., \& Wenske, V. 1993, A\&A, 268, 653

Petit, P., Donati, J.-F., \& Collier Cameron, A. 2002, MNRAS, 334, 374

Reiners, A. 2003, A\&A, 408, 707

Reiners, A., \& Schmitt, J. H. M. M. 2002a, A\&A, 384, 155

Reiners, A., \& Schmitt, J. H. M. M. 2002b, A\&A, 388, 1120

Reiners, A., \& Schmitt, J. H. M. M. 2003a, A\&A, 398, 647

Reiners, A., \& Schmitt, J. H. M. M. 2003b, A\&A, 412, 813

Reiners, A., \& Royer, F. 2004, A\&A, 415, 325

Reiners, A., Hünsch, M., Hempel, M., \& Schmitt, J. H. M. M. 2005, A\&A, 430, L17

Schrijver, C. J., \& Title, A. M. 2001, ApJ, 551, 1099

Schrijver, C. J., \& Zwaan, C. 2000, Solar and Magnetic Activity (Cambridge University Press)

Schou, J. 1998, ApJ, 122, 293

Siess, L., Dufour, E., \& Forestini, M. 2000, A\&A, 358, 593

Solanki, S. K. 1991, RvMA, 4, 208

Stahler, S. W., \& Palla, F. 2004, The Formation of Stars (Wiley-VCH)

Wolter, U., Schmitt, J. H. M. M., \& Van Wyk, F. 2005, A\&A, 436, 261 
A. Reiners: Differential rotation with temperature and rotation, Online Material $p 1$

\section{Online Material}




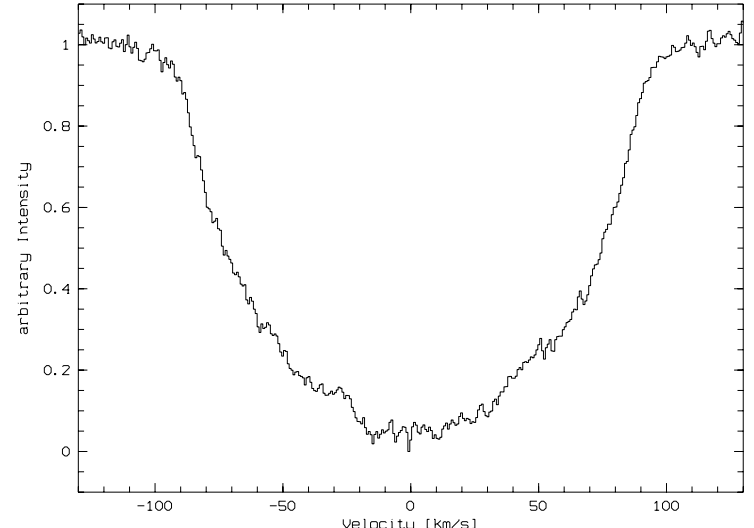

Fig. A.1. Broadening function of HD 307938. This object has also been studied with Doppler Imaging by Marsden et al. (2005).

\section{Appendix A: The first direct comparison to Doppler Imaging}

Recently, Marsden et al. (2005) have presented Doppler Images of HD 307938, a young active G dwarf in IC 2602. This star was also observed for this project in the FLAMES/UVES campaign, and I report on the rotation law in Table B.2. Marsden et al. (2005) took a time series over four nights detecting spectroscopic variability. Their data is contaminated by a significant amount of sunlight reflected by the moon, which they have carefully removed before constructing Doppler Images. The result of Doppler Imaging is that HD 307938 has a cool polar cap extending down to $\sim 60^{\circ}$ latitude, and $v \sin i=92 \pm 0.5 \mathrm{~km}^{-1}$, which excellently corresponds to $v \sin i=93.7 \pm 4.7 \mathrm{~km} \mathrm{~s}^{-1}$, as derived here (on comments about uncertainties in $v \sin i$, see Sect. 6). They also report marginal differential rotation of $\Delta \Omega=0.025 \pm 0.015 \mathrm{rad} \mathrm{d}^{-1}$ with a $1 \sigma$ error. From this result, one expects a value of $q_{2} / q_{1}$ that is only marginally less than 1.76 , the value for rigid rotation and the best guess for the limb darkening parameter. The cool polar spot found on HD 307938 influences $q_{2} / q_{1}$ as well, as it enlarges $q_{2} / q_{1}$ much more than the small deviation from rigid rotation does; $q_{2} / q_{1}$ is thus expected to be larger than 1.76. The spectrum secured for the analysis presented here is not contaminated by sunlight, and the broadening function derived is shown in Fig. A.1. The profile does not reveal large asymmetry, although a spot may be visible around $v \approx-30 \mathrm{~km} \mathrm{~s}^{-1}$. The profile is fully consistent with the broadening functions presented in Marsden et al. (2005), who were able to show temporal variations in the profile from lower quality data. The profile parameter determined from FTM is $q_{2} / q_{1}=1.78 \pm 0.01$, indicating no signs of solar-like differential rotation that is large enough to be detected with this method. However, the fact that $q_{2} / q_{1}$ is slightly larger than 1.76 supports the idea that a cool spot occupies the polar caps. Thus, the finding of marginal differential rotation and a cool polar spot is consistent with the result derived from FTM (besides the good consistency in $v \sin i$ ). This is the first time that a direct comparison of the results is possible, since the majority of Doppler Imaging targets usually show spot signatures that are stronger than what can be dealt with using FTM.
Appendix B: Tables of stars with measured differential rotation 
A. Reiners: Differential rotation with temperature and rotation, Online Material p 3

Table B.1. Field stars with measured differential rotation.

\begin{tabular}{|c|c|c|c|c|c|c|c|c|c|c|}
\hline Star & $\overline{\mathrm{HR}}$ & $\begin{array}{r}v \sin i \\
{\left[\mathrm{~km} \mathrm{~s}^{-1}\right]}\end{array}$ & $\begin{array}{c}\delta v \sin i \\
{\left[\mathrm{~km} \mathrm{~s}^{-1}\right]}\end{array}$ & $\overline{q q_{2} / q_{1}}$ & $\overline{c \delta q_{2} / q_{1}}$ & $\begin{array}{c}\Delta \Omega \\
\operatorname{rad~d}^{-1}\end{array}$ & $\begin{array}{c}\delta \Delta \Omega \\
\operatorname{rad~d}^{-1}\end{array}$ & $\begin{array}{c}T_{\text {eff }} \\
\mathrm{K}\end{array}$ & $\begin{array}{c}M_{V} \\
\mathrm{mag}\end{array}$ & $\begin{array}{c}P / \sin i \\
\mathrm{~d}\end{array}$ \\
\hline HD 432 & 21 & 71.0 & 3.6 & 1.78 & 0.03 & 0.00 & & 6763 & 0.96 & 3.08 \\
\hline HD 4089 & 187 & 23.5 & 1.2 & 1.82 & 0.02 & 0.00 & & 6161 & 2.75 & 5.01 \\
\hline HD 4247 & 197 & 42.7 & 2.1 & 1.77 & 0.04 & 0.00 & & 6825 & 3.08 & 1.90 \\
\hline HD 4757 & 230 & 93.8 & 4.7 & 1.73 & 0.03 & 0.00 & & 6706 & 1.34 & 2.00 \\
\hline HD 6706 & 329 & 46.0 & 2.3 & 1.83 & 0.06 & 0.00 & & 6551 & 2.77 & 2.21 \\
\hline HD 6903 & 339 & 87.7 & 4.4 & 1.69 & 0.02 & 0.54 & 0.534 & 6273 & 3.38 & 0.96 \\
\hline HD 15524 & 728 & 59.8 & 3.0 & 1.81 & 0.08 & 0.00 & & 6592 & 2.44 & 1.95 \\
\hline HD 17094 & 813 & 45.1 & 2.3 & 1.49 & 0.02 & 0.86 & 0.231 & 7141 & 2.17 & 2.50 \\
\hline HD 17206 & 818 & 25.6 & 1.3 & 1.70 & 0.02 & 0.15 & 0.184 & 6371 & 3.68 & 2.78 \\
\hline HD 18256 & 869 & 17.2 & 0.9 & 1.71 & 0.04 & 0.05 & 0.121 & 6332 & 2.93 & 5.91 \\
\hline HD 22001 & 1083 & 13.2 & 0.7 & 1.83 & 0.07 & 0.00 & & 6601 & 2.98 & 6.88 \\
\hline HD 22701 & 1107 & 55.0 & 2.8 & 1.83 & 0.03 & 0.00 & & 6610 & 2.70 & 1.88 \\
\hline HD 23754 & 1173 & 13.8 & 0.7 & 1.87 & 0.05 & 0.00 & & 6518 & 2.98 & 6.75 \\
\hline HD 24357 & 1201 & 65.8 & 3.3 & 1.82 & 0.05 & 0.00 & & 6895 & 2.83 & 1.35 \\
\hline HD 25457 & 1249 & 18.0 & 0.9 & 1.71 & 0.02 & 0.09 & 0.152 & 6333 & 4.07 & 3.35 \\
\hline HD 25621 & 1257 & 16.7 & 0.8 & 1.73 & 0.03 & 0.00 & & 6091 & 2.26 & 9.02 \\
\hline HD 27459 & 1356 & 78.3 & 3.9 & 1.78 & 0.05 & 0.00 & & 7642 & 2.15 & 1.29 \\
\hline HD 28677 & 1432 & 134.8 & 6.7 & 1.77 & 0.03 & 0.00 & & 6981 & 2.76 & 0.67 \\
\hline HD 28704 & 1434 & 88.1 & 4.4 & 1.79 & 0.03 & 0.00 & & 6672 & 2.43 & 1.30 \\
\hline HD 29875 & 1502 & 47.8 & 2.4 & 1.82 & 0.06 & 0.00 & & 7080 & 3.39 & 1.37 \\
\hline HD 29992 & 1503 & 97.5 & 4.9 & 1.75 & 0.04 & 0.00 & & 6742 & 2.64 & 1.04 \\
\hline HD 30034 & 1507 & 103.6 & 5.2 & 1.82 & 0.06 & 0.00 & & 7484 & 2.34 & 0.92 \\
\hline HD 30652 & 1543 & 17.3 & 0.9 & 1.78 & 0.03 & 0.00 & & 6408 & 3.59 & 4.23 \\
\hline HD 33167 & 1668 & 47.5 & 2.4 & 1.82 & 0.03 & 0.00 & & 6493 & 2.10 & 2.97 \\
\hline HD 35296 & 1780 & 15.9 & 0.8 & 1.75 & 0.02 & 0.00 & & 6060 & 4.11 & 4.09 \\
\hline HD 37147 & 1905 & 109.9 & 5.5 & 1.77 & 0.07 & 0.00 & & 7621 & 2.25 & 0.88 \\
\hline HD 41074 & 2132 & 87.8 & 4.4 & 1.78 & 0.06 & 0.00 & & 6912 & 2.45 & 1.20 \\
\hline HD 43386 & 2241 & 19.5 & 1.0 & 1.83 & 0.03 & 0.00 & & 6512 & 3.50 & 3.77 \\
\hline HD 44497 & 2287 & 89.4 & 4.5 & 1.79 & 0.03 & 0.00 & & 7010 & 1.79 & 1.56 \\
\hline HD 46273 & 2384 & 106.9 & 5.3 & 1.74 & 0.05 & 0.00 & & 6674 & 2.10 & 1.25 \\
\hline HD 48737 & 2484 & 66.1 & 3.3 & 1.78 & 0.04 & 0.00 & & 6496 & 2.32 & 1.93 \\
\hline HD 51199 & 2590 & 91.7 & 4.6 & 1.77 & 0.04 & 0.00 & & 6730 & 2.06 & 1.45 \\
\hline HD 55052 & 2706 & 81.8 & 4.1 & 1.80 & 0.04 & 0.00 & & 6668 & 0.63 & 3.21 \\
\hline HD 56986 & 2777 & 129.7 & 6.5 & 1.75 & 0.08 & 0.00 & & 6837 & 1.95 & 1.05 \\
\hline HD 57927 & 2816 & 89.5 & 4.5 & 1.75 & 0.02 & 0.00 & & 6772 & 1.40 & 1.99 \\
\hline HD 58579 & 2837 & 147.1 & 7.4 & 1.81 & 0.03 & 0.00 & & 7053 & 1.69 & 0.98 \\
\hline HD 58946 & 2852 & 59.0 & 3.0 & 1.76 & 0.07 & 0.00 & & 6892 & 2.79 & 1.54 \\
\hline HD 60111 & 2887 & 117.5 & 5.9 & 1.80 & 0.05 & 0.00 & & 7181 & 3.01 & 0.65 \\
\hline HD 61035 & 2926 & 124.2 & 6.2 & 1.80 & 0.03 & 0.00 & & 6986 & 3.09 & 0.62 \\
\hline HD 61110 & 2930 & 91.1 & 4.6 & 1.78 & 0.04 & 0.00 & & 6575 & 1.51 & 1.98 \\
\hline HD 62952 & 3015 & 127.5 & 6.4 & 1.80 & 0.08 & 0.00 & & 6933 & 1.53 & 1.26 \\
\hline HD 64685 & 3087 & 47.6 & 2.4 & 1.88 & 0.03 & 0.00 & & 6838 & 3.04 & 1.73 \\
\hline HD 67483 & 3184 & 52.4 & 4.1 & 1.50 & 0.07 & 0.69 & 0.279 & 6209 & 2.07 & 3.01 \\
\hline HD 69548 & 3254 & 53.9 & 2.7 & 1.77 & 0.05 & 0.00 & & 6705 & 3.53 & 1.27 \\
\hline HD 70958 & 3297 & 45.5 & 2.3 & 1.74 & 0.05 & 0.00 & & 6230 & 3.53 & 1.76 \\
\hline HD 70958 & 3297 & 46.1 & 2.3 & 1.75 & 0.01 & 0.00 & & 6230 & 3.53 & 1.74 \\
\hline HD 72943 & 3394 & 56.8 & 2.8 & 1.70 & 0.06 & 0.17 & 0.361 & 6897 & 1.81 & 2.51 \\
\hline HD 75486 & 3505 & 128.1 & 6.4 & 1.79 & 0.02 & 0.00 & & 6993 & 1.22 & 1.42 \\
\hline HD 76143 & 3537 & 83.0 & 4.2 & 1.79 & 0.03 & 0.00 & & 6579 & 1.58 & 2.10 \\
\hline HD 76582 & 3565 & 90.5 & 4.5 & 1.80 & 0.05 & 0.00 & & 7884 & 2.58 & 0.87 \\
\hline HD 77370 & 3598 & 60.4 & 3.0 & 1.71 & 0.03 & 0.20 & 0.408 & 6609 & 2.97 & 1.51 \\
\hline HD 77601 & 3603 & 140.7 & 7.0 & 1.82 & 0.02 & 0.00 & & 6421 & 0.44 & 2.21 \\
\hline HD 79940 & 3684 & 117.2 & 5.9 & 1.78 & 0.03 & 0.00 & & 6397 & 0.88 & 2.18 \\
\hline HD 81997 & 3759 & 30.4 & 1.5 & 1.73 & 0.01 & 0.00 & & 6471 & 3.28 & 2.72 \\
\hline HD 82554 & 3795 & 129.7 & 6.5 & 1.63 & 0.05 & 0.66 & 0.460 & 6272 & 1.32 & 1.68 \\
\hline HD 83287 & 3829 & 102.5 & 5.1 & 1.77 & 0.08 & 0.00 & & 7815 & 2.69 & 0.74 \\
\hline HD 83962 & 3859 & 140.3 & 7.0 & 1.72 & 0.08 & 0.00 & & 6507 & 1.53 & 1.30 \\
\hline HD 84607 & 3879 & 93.1 & 4.7 & 1.79 & 0.01 & 0.00 & & 7000 & 1.78 & 1.51 \\
\hline HD 88215 & 3991 & 97.5 & 4.9 & 1.78 & 0.05 & 0.00 & & 6823 & 3.04 & 0.85 \\
\hline HD 89254 & 4042 & 63.5 & 3.2 & 1.81 & 0.02 & 0.00 & & 7173 & 2.42 & 1.57 \\
\hline
\end{tabular}


Table B.1. continued.

\begin{tabular}{|c|c|c|c|c|c|c|c|c|c|c|}
\hline$\overline{\text { Star }}$ & 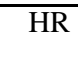 & $\begin{array}{r}v \sin i \\
{\left[\mathrm{~km} \mathrm{~s}^{-1}\right]}\end{array}$ & $\begin{array}{r}\delta v \sin i \\
{\left[\mathrm{~km} \mathrm{~s}^{-1}\right]}\end{array}$ & $q_{2} / q_{1}$ & $\bar{c} \delta q_{2} / q_{1}$ & $\begin{array}{c}\Delta \Omega \\
\operatorname{rad~d}^{-1}\end{array}$ & $\begin{array}{c}\delta \Delta \Omega \\
\operatorname{rad~d}^{-1}\end{array}$ & $\begin{array}{c}T_{\text {eff }} \\
\mathrm{K}\end{array}$ & $\begin{array}{c}M_{V} \\
\text { mag }\end{array}$ & $\begin{array}{c}P / \sin i \\
\mathrm{~d}\end{array}$ \\
\hline HD 89449 & 4054 & 17.3 & 1.7 & 1.44 & 0.04 & 0.45 & 0.127 & 6398 & 3.05 & 5.44 \\
\hline HD 89569 & 4061 & 12.2 & 0.7 & 1.57 & 0.02 & 0.21 & 0.070 & 6290 & 3.10 & 7.83 \\
\hline HD 89571 & 4062 & 133.9 & 6.7 & 1.79 & 0.07 & 0.00 & & & & \\
\hline HD 90089 & 4084 & 56.2 & 2.8 & 1.70 & 0.09 & 0.35 & 0.987 & 6674 & 3.60 & 1.19 \\
\hline HD 90589 & 4102 & 51.6 & 2.6 & 1.93 & 0.04 & 0.00 & & 6794 & 2.95 & 1.68 \\
\hline HD 96202 & 4314 & 93.4 & 4.7 & 1.77 & 0.05 & 0.00 & & 6747 & 2.34 & 1.25 \\
\hline HD 99329 & 4410 & 137.9 & 6.9 & 1.73 & 0.04 & 0.00 & & 6990 & 2.66 & 0.68 \\
\hline HD 100563 & 4455 & 13.5 & 0.7 & 1.67 & 0.04 & 0.14 & 0.134 & 6489 & 3.61 & 5.24 \\
\hline HD 105452 & 4623 & 23.5 & 1.2 & 1.59 & 0.02 & 0.40 & 0.151 & 6839 & 2.98 & 3.60 \\
\hline HD 106022 & 4642 & 77.2 & 3.9 & 1.86 & 0.06 & 0.00 & & 6651 & 2.28 & 1.60 \\
\hline HD 107326 & 4694 & 132.2 & 6.6 & 1.80 & 0.05 & 0.00 & & 7107 & 2.08 & 0.90 \\
\hline HD 108722 & 4753 & 97.0 & 4.8 & 1.78 & 0.04 & 0.00 & & 6490 & 1.71 & 1.74 \\
\hline HD 109085 & 4775 & 60.0 & 3.0 & 1.75 & 0.05 & 0.00 & & 6813 & 3.13 & 1.32 \\
\hline HD 109141 & 4776 & 135.7 & 6.8 & 1.79 & 0.03 & 0.00 & & 6881 & 2.84 & 0.66 \\
\hline HD 110385 & 4827 & 105.2 & 5.3 & 1.75 & 0.01 & 0.00 & & 6717 & 1.71 & 1.49 \\
\hline HD 110834 & 4843 & 133.3 & 6.7 & 1.73 & 0.06 & 0.00 & & 6244 & 0.99 & 1.93 \\
\hline HD 111812 & 4883 & 63.0 & 3.2 & 1.78 & 0.05 & 0.00 & & 5623 & 2.87 & 2.22 \\
\hline HD 112429 & 4916 & 119.6 & 6.0 & 1.77 & 0.03 & 0.00 & & 7126 & 2.97 & 0.66 \\
\hline HD 114378 & 4968 & 19.9 & 1.0 & 1.76 & 0.01 & 0.00 & & 6324 & 3.82 & 3.40 \\
\hline HD 115810 & 5025 & 99.2 & 5.0 & 1.82 & 0.06 & 0.00 & & 7185 & 1.95 & 1.24 \\
\hline HD 116568 & 5050 & 36.8 & 1.8 & 1.73 & 0.01 & 0.00 & & 6485 & 3.20 & 2.32 \\
\hline HD 118889 & 5138 & 140.6 & 7.0 & 1.82 & 0.06 & 0.00 & & 6951 & 2.40 & 0.76 \\
\hline HD 119756 & 5168 & 63.9 & 3.2 & 1.78 & 0.03 & 0.00 & & 6809 & 3.06 & 1.29 \\
\hline HD 120136 & 5185 & 15.6 & 1.0 & 1.57 & 0.04 & 0.31 & 0.134 & 6437 & 3.38 & 5.13 \\
\hline HD 121370 & 5235 & 13.5 & 1.3 & 1.46 & 0.03 & 0.21 & 0.059 & 6024 & 2.36 & 0.90 \\
\hline HD 122066 & 5257 & 40.6 & 2.0 & 1.81 & 0.01 & 0.00 & & 6395 & 2.18 & 3.45 \\
\hline HD 124780 & 5337 & 70.7 & 3.5 & 1.80 & 0.04 & 0.00 & & 7204 & 2.21 & 1.54 \\
\hline HD 124850 & 5338 & 15.0 & 0.8 & 1.91 & 0.04 & 0.00 & & 6075 & 2.85 & 7.71 \\
\hline HD 127739 & 5434 & 55.8 & 2.8 & 1.81 & 0.01 & 0.00 & & 6787 & 2.20 & 2.20 \\
\hline HD 127821 & 5436 & 55.6 & 2.8 & 1.75 & 0.04 & 0.00 & & 6601 & 3.76 & 1.14 \\
\hline HD 129153 & 5473 & 105.7 & 5.3 & 1.78 & 0.06 & 0.00 & & 7693 & 2.70 & 0.73 \\
\hline HD 129502 & 5487 & 47.0 & 2.4 & 1.80 & 0.03 & 0.00 & & 6695 & 2.94 & 1.91 \\
\hline HD 129926 & 5497 & 112.5 & 5.6 & 1.74 & 0.03 & 0.00 & & 6048 & 3.91 & 0.64 \\
\hline HD 132052 & 5570 & 113.2 & 5.7 & 1.77 & 0.06 & 0.00 & & 6964 & 2.19 & 1.03 \\
\hline HD 136359 & 5700 & 20.3 & 1.0 & 1.78 & 0.01 & 0.00 & & 6296 & 3.02 & 4.86 \\
\hline HD 136751 & 5716 & 72.7 & 3.6 & 1.77 & 0.02 & 0.00 & & 6810 & 2.31 & 1.60 \\
\hline HD 138917 & 5788 & 85.8 & 5.4 & 1.99 & 0.06 & 0.00 & & & & \\
\hline HD 139225 & 5804 & 104.4 & 5.2 & 1.76 & 0.03 & 0.00 & & 6960 & 2.50 & 0.97 \\
\hline HD 139664 & 5825 & 71.6 & 3.6 & 1.77 & 0.05 & 0.00 & & 6681 & 3.57 & 0.94 \\
\hline HD 142908 & 5936 & 75.8 & 3.8 & 1.80 & 0.05 & 0.00 & & 6849 & 2.33 & 1.50 \\
\hline HD 143466 & 5960 & 141.3 & 7.1 & 1.77 & 0.05 & 0.00 & & 7235 & 2.20 & 0.77 \\
\hline HD 147365 & 6091 & 72.5 & 3.6 & 1.77 & 0.06 & 0.00 & & 6657 & 3.46 & 0.99 \\
\hline HD 147449 & 6093 & 76.4 & 3.8 & 1.79 & 0.04 & 0.00 & & 6973 & 2.70 & 1.21 \\
\hline HD 147449 & 6093 & 77.1 & 3.9 & 1.80 & 0.03 & 0.00 & & 6973 & 2.70 & 1.20 \\
\hline HD 148048 & 6116 & 84.8 & 4.2 & 1.78 & 0.05 & 0.00 & & 6731 & 2.43 & 1.32 \\
\hline HD 150557 & 6205 & 61.8 & 3.1 & 1.83 & 0.04 & 0.00 & & 6959 & 2.01 & 2.06 \\
\hline HD 151613 & 6237 & 47.5 & 2.4 & 1.84 & 0.07 & 0.00 & & 6630 & 2.80 & 2.06 \\
\hline HD 155103 & 6377 & 57.9 & 2.9 & 1.81 & 0.05 & 0.00 & & 7150 & 2.55 & 1.63 \\
\hline HD 156295 & 6421 & 107.4 & 5.4 & 1.79 & 0.07 & 0.00 & & 7818 & 2.56 & 0.75 \\
\hline HD 160915 & 6595 & 12.4 & 0.6 & 1.60 & 0.03 & 0.23 & 0.101 & 6356 & 3.58 & 6.07 \\
\hline HD 164259 & 6710 & 69.3 & 3.5 & 1.75 & 0.04 & 0.00 & & 6704 & 2.70 & 1.44 \\
\hline HD 165373 & 6754 & 79.9 & 4.0 & 1.74 & 0.03 & 0.00 & & 6976 & 2.08 & 1.54 \\
\hline HD 171834 & 6987 & 71.3 & 3.6 & 1.77 & 0.04 & 0.00 & & 6622 & 2.50 & 1.58 \\
\hline HD 173417 & 7044 & 53.9 & 2.7 & 1.83 & 0.04 & 0.00 & & 6780 & 1.81 & 2.74 \\
\hline HD 173667 & 7061 & 18.0 & 2.0 & 1.40 & 0.02 & 0.44 & 0.104 & 6363 & 2.78 & 5.99 \\
\hline HD 175317 & 7126 & 17.1 & 0.9 & 1.58 & 0.04 & 0.30 & 0.136 & 6563 & 3.11 & 5.07 \\
\hline HD 175824 & 7154 & 53.7 & 2.7 & 1.69 & 0.03 & 0.15 & 0.179 & 6232 & 1.72 & 3.43 \\
\hline HD 182640 & 7377 & 87.3 & 4.4 & 1.67 & 0.06 & 0.62 & 0.753 & 7016 & 2.46 & 1.17 \\
\hline HD 185124 & 7460 & 87.0 & 4.4 & 1.71 & 0.03 & 0.30 & 0.604 & 6680 & 2.98 & 1.02 \\
\hline HD 186005 & 7489 & 149.9 & 7.5 & 1.79 & 0.04 & 0.00 & & 6988 & 1.68 & 0.98 \\
\hline
\end{tabular}


A. Reiners: Differential rotation with temperature and rotation, Online Material p 5

Table B.1. continued.

\begin{tabular}{crrrrrrrrrr}
\hline \hline Star & HR & $\begin{array}{r}v \sin i \\
{\left[\mathrm{~km} \mathrm{~s}^{-1}\right]}\end{array}$ & $\begin{array}{r}\delta v \sin i \\
{\left[\mathrm{~km} \mathrm{~s}^{-1}\right]}\end{array}$ & $q_{2} / q_{1}$ & $\delta q_{2} / q_{1}$ & $\begin{array}{c}\Delta \Omega \\
\mathrm{rad} \mathrm{d}^{-1}\end{array}$ & $\begin{array}{c}\delta \Delta \Omega \\
\mathrm{rad} \mathrm{d}^{-1}\end{array}$ & $\begin{array}{c}T_{\text {eff }} \\
\mathrm{K}\end{array}$ & $\begin{array}{c}M_{V} \\
\mathrm{mag}\end{array}$ & $\begin{array}{c}P / \sin i \\
\mathrm{~d}\end{array}$ \\
\hline HD 187532 & 7553 & 77.5 & 3.9 & 1.75 & 0.03 & 0.00 & & 6788 & 3.34 & 0.94 \\
HD 189245 & 7631 & 72.6 & 3.6 & 1.74 & 0.03 & 0.00 & & 6259 & 4.06 & 0.85 \\
HD 190004 & 7657 & 136.1 & 6.8 & 1.80 & 0.04 & 0.00 & & 6974 & 2.47 & 0.76 \\
HD 197692 & 7936 & 41.7 & 2.1 & 1.62 & 0.02 & 0.65 & 0.286 & 6587 & 3.33 & 1.86 \\
HD 199260 & 8013 & 13.7 & 0.7 & 1.79 & 0.03 & 0.00 & & 6213 & 4.18 & 4.36 \\
HD 201636 & 8099 & 58.8 & 2.9 & 1.86 & 0.03 & 0.00 & & 6700 & 2.20 & 2.15 \\
HD 203925 & 8198 & 70.7 & 3.5 & 1.80 & 0.03 & 0.00 & & 6845 & 1.15 & 2.77 \\
HD 205289 & 8245 & 57.5 & 2.9 & 1.74 & 0.03 & 0.00 & & 6525 & 3.24 & 1.44 \\
HD 206043 & 8276 & 134.0 & 6.7 & 1.74 & 0.04 & 0.00 & & 7092 & 2.87 & 0.62 \\
HD 207958 & 8351 & 69.3 & 3.5 & 1.84 & 0.03 & 0.00 & & 6747 & 2.97 & 1.26 \\
HD 210302 & 8447 & 13.6 & 0.7 & 1.72 & 0.04 & 0.00 & & 6465 & 3.52 & 5.46 \\
HD 210459 & 8454 & 147.4 & 7.4 & 1.76 & 0.03 & 0.00 & & 6376 & 0.21 & 2.89 \\
HD 213051 & 8558 & 48.4 & 2.4 & 1.85 & 0.08 & 0.00 & & & & \\
HD 213845 & 8592 & 35.7 & 1.8 & 1.71 & 0.02 & 0.14 & 0.234 & 6551 & 3.35 & 2.18 \\
HD 219693 & 8859 & 19.9 & 1.0 & 1.81 & 0.01 & 0.00 & & 6461 & 2.82 & 5.14 \\
HD 220657 & 8905 & 73.4 & 3.7 & 1.74 & 0.03 & 0.00 & & 5801 & 2.41 & 2.15 \\
\hline
\end{tabular}

Table B.2. Stars in cluster fields, FLAMES/UVES observations.

\begin{tabular}{ccrrrrrrrrc}
\hline \hline Star & Cluster & $\begin{array}{r}v \sin i \\
{\left[\mathrm{~km} \mathrm{~s}^{-1}\right]}\end{array}$ & $\begin{array}{r}\delta v \sin i \\
{\left[\mathrm{~km} \mathrm{~s}^{-1}\right]}\end{array}$ & $\begin{array}{c}q_{2} / q_{1} \\
\end{array}$ & $\delta q_{2} / q_{1}$ & $\begin{array}{c}\Delta \Omega \\
\mathrm{rad} \mathrm{d}^{-1}\end{array}$ & $\begin{array}{c}\delta \Delta \Omega \\
\mathrm{rad} \mathrm{d}^{-1}\end{array}$ & $\begin{array}{c}T_{\text {eff }} \\
\mathrm{K}\end{array}$ & $\begin{array}{c}M_{V} \\
\mathrm{mag}\end{array}$ & $\begin{array}{c}P / \sin i \\
\mathrm{~d}\end{array}$ \\
\hline NGC 6475 69 & NGC 6475 & 94.9 & 4.7 & 1.76 & 0.01 & 0.00 & & 6822 & 3.27 & 0.75 \\
NGC 6475 41 & NGC 6475 & 80.1 & 4.0 & 1.71 & 0.01 & 0.41 & 0.497 & 6346 & 3.86 & 0.76 \\
BD+20 2161 & Praesepe & 72.7 & 3.6 & 1.76 & 0.01 & 0.00 & & 6864 & 3.23 & 0.99 \\
BD+20 2170 & Praesepe & 94.4 & 4.7 & 1.71 & 0.01 & 0.46 & 0.557 & 6484 & 3.69 & 0.68 \\
Cl* NGC 2632 KW 230 $^{*}$ Praesepe & 78.9 & 3.9 & 1.75 & 0.01 & 0.00 & & 6628 & 3.51 & 0.85 \\
Cl* IC 2391 L 33 & IC 2391 & 81.1 & 4.1 & 1.74 & 0.01 & 0.00 & & 6414 & 3.78 & 0.77 \\
HD 307938 & IC 2602 & 93.7 & 4.7 & 1.78 & 0.01 & 0.00 & & 5743 & 4.64 & 0.51 \\
HD 308012 & IC 2602 & 45.7 & 2.3 & 1.70 & 0.01 & 0.36 & 0.306 & 6147 & 4.12 & 1.23 \\
Cl* IC 4665 V 69 & IC 4665 & 45.1 & 2.3 & 1.74 & 0.01 & 0.00 & & 6180 & 4.08 & 1.26 \\
Cl* IC 4665 V 102 & IC 4665 & 105.0 & 12.0 & 1.39 & 0.01 & 3.62 & 0.776 & 7136 & 2.93 & 0.73 \\
Cl* IC 4665 V 97 & IC 4665 & 52.0 & 2.6 & 1.90 & 0.01 & 0.00 & & 6628 & 3.51 & 1.29 \\
\hline
\end{tabular}

